\title{
Higher-order supersymmetric quantum mechanics
}

\author{
David J. Fernández C. and Nicolás Fernández-García \\ Departamento de Física, CINVESTAV \\ AP 14-740, 07000 México DF, Mexico
}

\begin{abstract}
We review the higher-order supersymmetric quantum mechanics (H-SUSY QM), which involves differential intertwining operators of order greater than one. The iterations of first-order SUSY transformations are used to derive in a simple way the higher-order case. The second order technique is addressed directly, and through this approach unexpected possibilities for designing spectra are uncovered. The formalism is applied to the harmonic oscillator: the corresponding H-SUSY partner Hamiltonians are ruled by polynomial Heisenberg algebras which allow a straight construction of the coherent states.
\end{abstract}




\section{Contents}

$\begin{array}{lll}1 & \text { Introduction } & 3\end{array}$

2 Higher-order supersvmmetric quantum mechanics: iterative approach 5

3 Higher-order supersvmmetric quantum mechanics: direct approach 11

3.1 Second-order supersvmmetric quantum mechanics . . . . . . . . . . . . . . 11

3.2 Classification of the second-order SUSY transformations . . . . . . . . . . 13

3.2 .1 The real case with $c>0 \ldots \ldots \ldots \ldots \ldots$

3.2 .2 The confluent case with $c=0 \ldots \ldots \ldots \ldots$

3.2 .3 The complex case with $c<0 \ldots \ldots \ldots \ldots$

4 An example: the harmonic oscillator 20

4.1 Polvnomial deformations of the Heisenberg algebra . . . . . . . . . . . 20

4.2 SUSY partners of the oscillator . . . . . . . . . . . . . . . . . . . . . 22

4.2.1 The Abraham-Moses potentials . . . . . . . . . . . . . . . . . 23

4.3 Non-linear algebra of $H_{b} \ldots \ldots \ldots \ldots \ldots \ldots \ldots$

4.4 Linearization of the non-linear algebra of $H_{b} \ldots \ldots \ldots . \ldots 26$

5 Coherent states for the SUSY partners of the oscillator 29

5.1 Standard coherent states . . . . . . . . . . . . . . . . . . . . . . . 29

5.2 Coherent states for $H_{4} \quad \ldots \ldots \ldots \ldots \ldots$

$\begin{array}{lll}6 & \text { Conclusions and outlook } & 37\end{array}$ 


\section{Introduction}

The number of exactly solvable problems in non-relativistic quantum mechanics is small, and most of them can be dealt with the factorization method. This technique, introduced long ago by Schrödinger [1,2], was analyzed in depth by Infeld and Hull [3], who made an exhaustive classification of factorizable potentials. Later on, Witten noticed the possibility of arranging the Schrödinger's Hamiltonians into isospectral pairs (supersymmetric partners) [4]. The resulting supersymmetric quantum mechanics (SUSY QM) revived the study of exactly solvable Hamiltonians (see e.g. [5]). An additional step was Mielnik's factorization through which the general SUSY partner for the oscillator for a certain factorization energy was found [6]; this technique was immediately applied to the hydrogen potential [7]. Meanwhile Nieto [8], Andrianov et al [9] and Sukumar [10-14] put the method on its natural background discovering the links between SUSY, factorization and Darboux algorithm, causing then a renaissance of the related algebraic methods [15-55] (for a recent review see [56] and references therein).

These procedures can be recovered from a general setting in which a first-order differential operator intertwines two Hamiltonians $[57,58]$. This so-called first-order intertwining technique suggests further generalizations: the most obvious one involves a $k$-th order differential intertwining operator and gives place to the higher-order supersymmetric quantum mechanics (H-SUSY QM) [59-77]. It can be achieved by iterations of first order SUSY transformations [64]. An alternative to deal with the problem consists in looking for the $k$-th order operator directly, expressing the intertwiner as a sum of the $k+1$ terms $g_{i}(x) d^{i} / d x^{i}, i=0, \ldots, k$, and solving the system of equations resulting from the intertwining relationship for the $g_{i}(x)$ 's. By assuming that the initial Hamiltonian is solvable, new solvable ones and their eigenstates can be generated through the two previous formulations.

Concerning applications, the procedure works successfully to generate $k$-parametric families of potentials almost isospectral to the harmonic oscillator [64,67], the radial hydrogenlike potentials [65,66], and in the free particle case [69] (for a collection of recent papers on SUSY QM see [78]). Specially interesting are the SUSY partners of the oscillator because through them some connections with other important subjects of mathematical physics can be established.

In the first place let us notice the existence of differential annihilation and creation operators of order greater than one for the SUSY partners of the oscillator. These operators provide natural realizations of the so called polynomial Heisenberg algebras [67,75,79-83]. It is interesting as well that these non-linear algebras admit partial linearizations [84-86], i.e., through appropriate modifications on the ladder operators the standard Heisenberg algebra is recovered on the subspace spanned by the eigenfunctions intertwined with the physical eigenstates of the oscillator.

In the second place let us mention the construction of coherent states (CS) for potentials generated through the intertwining technique [67, 82, 84-94]. In this direction Fukui and Aizawa have derived some CS for the 'shape invariant' potentials [87], a particular class of solvable potentials generated long ago by Infeld and Hull through the factorization method [3]. The first work involving CS for the simplest non-trivial family of potentials isospectral to the oscillator (Abraham-Moses [6,95]) was done in 1994 [84] (see also [85,86]). Later on 
various developments have appeared, e.g., some authors have constructed the CS for a class of anharmonic oscillators which spectra consist of a part isospectral to the oscillator plus one lower energy at a multiple of the spacing between the oscillator levels [91]. Furthermore, CS have been derived [82] for families of SUSY partner potentials having an arbitrary eigenvalue below the ground state energy of the oscillator $[11,43,64]$. Recently, it has been implemented as well the CS construction for the general H-SUSY partners of the oscillator [67].

In these lecture notes we are going to address the subjects mentioned above. We will start with the iterative H-SUSY QM to generate families of potentials (almost) isospectral to the initial one. Then, we will formulate the same problem directly, by assuming that the intertwining operator is of $k$-th order. Due to the difficulty involved in this problem, we just will illustrate the technique through the second-order supersymmetric quantum mechanics. Then we will apply these generation procedures to the harmonic oscillator. It will be shown that the intrinsic symmetry of the H-SUSY partners of the oscillator is generated by a pair of differential annihilation and creation operators of order greater than one, giving place to explicit representations of the polynomial Heisenberg algebras. We will show as well an interesting linearization procedure for the nonlinear algebras characteristic of the H-SUSY partners of the oscillator. We will perform then the coherent states construction in the nonlinear as well as in the linear cases. We will conclude these notes with some discussion on the future of SUSY QM. 


\section{Higher-order supersymmetric quantum mechanics: iterative approach}

Let us start with two Schrödinger Hamiltonians

$$
H_{i}=-\frac{1}{2} \frac{d^{2}}{d x^{2}}+V_{i}(x) \quad i=0,1
$$

and suppose the existence of a first order differential operator $A_{1}^{\dagger}$ intertwining them

$$
H_{1} A_{1}^{\dagger}=A_{1}^{\dagger} H_{0}
$$

where

$$
A_{1}^{\dagger}=\frac{1}{\sqrt{2}}\left(-\frac{d}{d x}+\alpha_{1}(x)\right)
$$

the superpotential $\alpha_{1}(x)$ being a real function to be determined. In order to 'pass' the differential operator $d / d x$ and its powers to the right in the terms arising from (2), we use the following operator relationships

$$
\begin{aligned}
& \frac{d}{d x} f=f \frac{d}{d x}+f^{\prime} \\
& \frac{d^{2}}{d x^{2}} f=f \frac{d^{2}}{d x^{2}}+2 f^{\prime} \frac{d}{d x}+f^{\prime \prime}
\end{aligned}
$$

Then, it is straightforward to show that

$$
\begin{aligned}
& \sqrt{2} H_{1} A_{1}^{\dagger}=\frac{1}{2} \frac{d^{3}}{d x^{3}}-\frac{\alpha_{1}}{2} \frac{d^{2}}{d x^{2}}-\left(V_{1}+\alpha_{1}^{\prime}\right) \frac{d}{d x}+\alpha_{1} V_{1}-\frac{\alpha_{1}^{\prime \prime}}{2} \\
& \sqrt{2} A_{1}^{\dagger} H_{0}=\frac{1}{2} \frac{d^{3}}{d x^{3}}-\frac{\alpha_{1}}{2} \frac{d^{2}}{d x^{2}}-V_{0} \frac{d}{d x}+\alpha_{1} V_{0}-V_{0}^{\prime}
\end{aligned}
$$

Due to (21) we make equal the coefficient of the same powers of $d / d x$ of the two previous equations to obtain

$$
\begin{aligned}
& V_{1}=V_{0}-\alpha_{1}^{\prime} \\
& \alpha_{1} V_{1}-\frac{\alpha_{1}^{\prime \prime}}{2}=\alpha_{1} V_{0}-V_{0}^{\prime}
\end{aligned}
$$

Substituting the expression for $V_{1}$ of (41) in (5) and integrating the result we get:

$$
\alpha_{1}^{\prime}+\alpha_{1}^{2}=2\left(V_{0}-\epsilon\right)
$$

From now on we are going to express explicitly the dependence of the superpotential in terms of the factorization energy $\epsilon$ in the way $\alpha_{1}(x, \epsilon)$. If a function $u^{(0)}(x)$ such that $\alpha_{1}(x, \epsilon)=u^{(0)^{\prime}} / u^{(0)}$ is used, we have that (46) become:

$$
\begin{aligned}
& -\frac{1}{2} u^{(0)^{\prime \prime}}+V_{0} u^{(0)}=\epsilon u^{(0)} \\
& V_{1}=V_{0}-\left(\frac{u^{(0)^{\prime}}}{u^{(0)}}\right)^{\prime}
\end{aligned}
$$


i.e., $u^{(0)}$ is a solution (not necessarily physical) of the initial stationary Schrödinger equation associated to $\epsilon$.

Let us notice that (46) guarantee that $H_{0}$ and $H_{1}$ become factorized:

$$
H_{0}=A_{1} A_{1}^{\dagger}+\epsilon \quad H_{1}=A_{1}^{\dagger} A_{1}+\epsilon
$$

where

$$
A_{1}=\frac{1}{\sqrt{2}}\left(\frac{d}{d x}+\alpha_{1}(x, \epsilon)\right)
$$

is the operator adjoint to $A_{1}^{\dagger}$.

Suppose that $V_{0}(x)$ is a solvable potential with eigenfunctions $\psi_{n}^{(0)}(x)$ and eigenvalues $E_{n}, n=0,1, \ldots$ Furthermore, let us assume that we have found a solution $\alpha_{1}\left(x, \epsilon_{1}\right)\left(u_{1}^{(0)}(x)\right)$ to the Riccati equation (6) (Schrödinger equation (7)) for a given value of the factorization energy $\epsilon=\epsilon_{1} \leq E_{0}$, where $E_{0}$ is the ground state energy of $H_{0}$. Thus, the $V_{1}(x)$ given in (4) is a completely determined solvable potential with normalized eigenfunctions

$$
\begin{aligned}
& \psi_{\epsilon_{1}}^{(1)}(x) \propto e^{-\int_{0}^{x} \alpha_{1}\left(y, \epsilon_{1}\right) d y}=\frac{1}{u_{1}^{(0)}(x)} \\
& \psi_{n}^{(1)}(x)=\frac{A_{1}^{\dagger} \psi_{n}^{(0)}(x)}{\sqrt{E_{n}-\epsilon_{1}}}
\end{aligned}
$$

and eigenvalues $\left\{\epsilon_{1}, E_{n}, n=0,1, \ldots\right\}$. Let us remark that the restriction $\epsilon_{1} \leq E_{0}$ is crucial to avoid the existence of singularities in $\alpha_{1}\left(x, \epsilon_{1}\right)$, in $V_{1}$ and also in the $\psi_{\epsilon_{1}}^{(1)}, \psi_{n}^{(1)}$ of (1112). Indeed, if $\epsilon_{1}$ would be greater than $E_{0}$, the transformation function $u_{1}^{(0)}$ would have always zeros in the initial $x$-domain and thus $\alpha_{1}\left(x, \epsilon_{1}\right)$ would have singularities at those points. If however $\epsilon_{1} \leq E_{0}$, then $u_{1}^{(0)}(x)$ will have at most one zero; by exploring the two-dimensional subspace of solutions associated to $\epsilon_{1}$ it is possible to find a subset of nodeless solutions (see e.g. $[10,11])$. By simplicity, we shall assume that the factorization energy used to generate any new Hamiltonian through the first-order SUSY is below the ground state energy of the initial Hamiltonian. We shall suppose as well that, for fixed $\epsilon_{1}$, the arbitrary parameter of a general Riccati solution (6) has been adjusted in order to avoid the singularities in the $\alpha$ 's.

Now we iterate the previous technique, taking $V_{1}(x)$ as the given solvable potential to generate a new one $V_{2}(x)$ through an intertwining operator $A_{2}^{\dagger}$ and a different factorization energy $\epsilon_{2}$, with $\epsilon_{2}<\epsilon_{1}$. The corresponding intertwining relationship, $H_{2} A_{2}^{\dagger}=A_{2}^{\dagger} H_{1}$, leads to equations similar to (46):

$$
\begin{aligned}
& \alpha_{2}^{\prime}\left(x, \epsilon_{2}\right)+\alpha_{2}^{2}\left(x, \epsilon_{2}\right)=2\left[V_{1}(x)-\epsilon_{2}\right] \\
& V_{2}(x)=V_{1}(x)-\alpha_{2}^{\prime}\left(x, \epsilon_{2}\right)
\end{aligned}
$$

In terms of transformation functions $u_{2}^{(1)}(x)$ such that $\alpha_{2}\left(x, \epsilon_{2}\right)=u_{2}^{(1)^{\prime}} / u_{2}^{(1)}$ we have:

$$
\begin{aligned}
& -\frac{1}{2} u_{2}^{(1)^{\prime \prime}}+V_{1} u_{2}^{(1)}=\epsilon_{2} u_{2}^{(1)} \\
& V_{2}=V_{1}-\left(\frac{u_{2}^{(1)^{\prime}}}{u_{2}^{(1)}}\right)^{\prime}
\end{aligned}
$$


An important result to be proved now is that the solutions to (13) can be algebraically determined through solutions of the initial Riccati equation (6) for the factorization energies $\epsilon_{1}$ and $\epsilon_{2}$ [64-69]. We stick to [96] because there the calculations are transparent. To find the corresponding formula [69], we know that the two initial Riccati solutions satisfy:

$$
\begin{aligned}
& \alpha_{1}^{\prime}\left(x, \epsilon_{1}\right)+\alpha_{1}^{2}\left(x, \epsilon_{1}\right)=2\left[V_{0}(x)-\epsilon_{1}\right] \\
& \alpha_{1}^{\prime}\left(x, \epsilon_{2}\right)+\alpha_{1}^{2}\left(x, \epsilon_{2}\right)=2\left[V_{0}(x)-\epsilon_{2}\right]
\end{aligned}
$$

At the Schrödinger level we have that

$$
\begin{aligned}
& H_{0} u_{1}^{(0)}(x)=\epsilon_{1} u_{1}^{(0)}(x) \\
& H_{0} u_{2}^{(0)}(x)=\epsilon_{2} u_{2}^{(0)}(x)
\end{aligned}
$$

where

$$
\begin{aligned}
& u_{1}^{(0)}(x) \propto e^{\int_{0}^{x} \alpha_{1}\left(y, \epsilon_{1}\right) d y} \\
& u_{2}^{(0)}(x) \propto e^{\int_{0}^{x} \alpha_{1}\left(y, \epsilon_{2}\right) d y}
\end{aligned}
$$

Let us remember that $u_{1}^{(0)}(x)$ is used to implement the first SUSY transformation and that the eigenfunction of $H_{1}$ associated to $\epsilon_{1}$ is given by (11). On the other hand, the eigenfunction of $H_{1}$ associated to $\epsilon_{2}$ is given by:

$$
u_{2}^{(1)} \propto A_{1}^{\dagger} u_{2}^{(0)} \propto-u_{2}^{(0)^{\prime}}+\alpha_{1}\left(x, \epsilon_{1}\right) u_{2}^{(0)} \propto \frac{W\left(u_{1}^{(0)}, u_{2}^{(0)}\right)}{u_{1}^{(0)}}
$$

and since that

$$
u_{2}^{(0)^{\prime}}=\alpha_{1}\left(x, \epsilon_{2}\right) u_{2}^{(0)}
$$

we get:

$$
u_{2}^{(1)} \propto\left[\alpha_{1}\left(x, \epsilon_{1}\right)-\alpha_{1}\left(x, \epsilon_{2}\right)\right] u_{2}^{(0)}
$$

In order to implement the second SUSY transformation, we express $u_{2}^{(1)}$ in the standard way in terms of the corresponding superpotential:

$$
u_{2}^{(1)}(x) \propto e^{\int_{0}^{x} \alpha_{2}\left(y, \epsilon_{2}\right) d y}
$$

By plugging (21) in (20) we arrive at

$$
e^{\int_{0}^{x} \alpha_{2}\left(y, \epsilon_{2}\right) d y} \propto\left[\alpha_{1}\left(x, \epsilon_{1}\right)-\alpha_{1}\left(x, \epsilon_{2}\right)\right] u_{2}^{(0)}
$$

By taking the logarithm of both sides of the previous equation:

$$
\int_{0}^{x} \alpha_{2}\left(y, \epsilon_{2}\right) d y=\ln u_{2}^{(0)}+\ln \left[\alpha_{1}\left(x, \epsilon_{1}\right)-\alpha_{1}\left(x, \epsilon_{2}\right)\right]+\text { constant }
$$


Deriving this expression with respect to $x$ :

$$
\alpha_{2}\left(x, \epsilon_{2}\right)=\alpha_{1}\left(x, \epsilon_{2}\right)+\frac{\alpha_{1}^{\prime}\left(x, \epsilon_{1}\right)-\alpha_{1}^{\prime}\left(x, \epsilon_{2}\right)}{\alpha_{1}\left(x, \epsilon_{1}\right)-\alpha_{1}\left(x, \epsilon_{2}\right)}
$$

By using the initial Riccati equations (17) we obtain:

$$
\frac{\alpha_{1}^{\prime}\left(x, \epsilon_{1}\right)-\alpha_{1}^{\prime}\left(x, \epsilon_{2}\right)}{\alpha_{1}\left(x, \epsilon_{1}\right)-\alpha_{1}\left(x, \epsilon_{2}\right)}=-\alpha_{1}\left(x, \epsilon_{2}\right)-\alpha_{1}\left(x, \epsilon_{1}\right)-\frac{2\left(\epsilon_{1}-\epsilon_{2}\right)}{\alpha_{1}\left(x, \epsilon_{1}\right)-\alpha_{1}\left(x, \epsilon_{2}\right)}
$$

Therefore

$$
\alpha_{2}\left(x, \epsilon_{2}\right)=-\alpha_{1}\left(x, \epsilon_{1}\right)-\frac{2\left(\epsilon_{1}-\epsilon_{2}\right)}{\alpha_{1}\left(x, \epsilon_{1}\right)-\alpha_{1}\left(x, \epsilon_{2}\right)}
$$

This formula expresses the solution to (13) with $V_{1}(x)=V_{0}(x)-\alpha_{1}^{\prime}\left(x, \epsilon_{1}\right)$ in form of a finite difference formula involving two solutions $\alpha_{1}\left(x, \epsilon_{1}\right), \alpha_{1}\left(x, \epsilon_{2}\right)$ of the Riccati equation (6) for the factorization energies $\epsilon_{1}, \epsilon_{2}$ (see also [64]). Notice that a similar formula has been used by Adler in order to discuss the Bäcklund transformations of the Painlevé equations [80]. The potential $V_{2}(x)$ reads:

$$
V_{2}(x)=V_{1}(x)-\alpha_{2}^{\prime}\left(x, \epsilon_{2}\right)=V_{0}(x)+\left[\frac{2\left(\epsilon_{1}-\epsilon_{2}\right)}{\alpha_{1}\left(x, \epsilon_{1}\right)-\alpha_{1}\left(x, \epsilon_{2}\right)}\right]^{\prime}
$$

The eigenfunctions associated to $H_{2}$ are given by:

$$
\begin{aligned}
& \psi_{\epsilon_{2}}^{(2)}(x) \propto e^{-\int_{0}^{x} \alpha_{2}\left(y, \epsilon_{2}\right) d y}=\frac{1}{u_{2}^{(1)}} \\
& \psi_{\epsilon_{1}}^{(2)}(x)=\frac{A_{2}^{\dagger} \psi_{\epsilon_{1}}^{(1)}(x)}{\sqrt{\epsilon_{1}-\epsilon_{2}}} \\
& \psi_{n}^{(2)}(x)=\frac{A_{2}^{\dagger} \psi_{n}^{(1)}(x)}{\sqrt{E_{n}-\epsilon_{2}}}=\frac{A_{2}^{\dagger} A_{1}^{\dagger} \psi_{n}^{(0)}(x)}{\sqrt{\left(E_{n}-\epsilon_{1}\right)\left(E_{n}-\epsilon_{2}\right)}}
\end{aligned}
$$

The corresponding eigenvalues are $\left\{\epsilon_{2}, \epsilon_{1}, E_{n}, n=0,1, \ldots\right\}$.

We can continue the iteration process as many times as solutions for different values $\epsilon_{i}$ to the initial Riccati equation (6) we have. Suppose that we know $k$ of these, $\left\{\alpha_{1}\left(x, \epsilon_{i}\right), i=\right.$ $\left.1, \ldots, k, \epsilon_{i+1}<\epsilon_{i}\right\}$, and we iterate the process $k$ times. Hence, a new solvable Hamiltonian $H_{k}$ will be gotten whose potential reads:

$$
V_{k}(x)=V_{k-1}(x)-\alpha_{k}^{\prime}\left(x, \epsilon_{k}\right)=V_{0}(x)-\sum_{i=1}^{k} \alpha_{i}^{\prime}\left(x, \epsilon_{i}\right)
$$

where $\alpha_{i}\left(x, \epsilon_{i}\right)$ is given by a recursive finite difference formula generalizing (26):

$$
\alpha_{i+1}\left(x, \epsilon_{i+1}\right)=-\alpha_{i}\left(x, \epsilon_{i}\right)-\frac{2\left(\epsilon_{i}-\epsilon_{i+1}\right)}{\alpha_{i}\left(x, \epsilon_{i}\right)-\alpha_{i}\left(x, \epsilon_{i+1}\right)} \quad i=1, \ldots, k-1
$$


The eigenfunctions are given by:

$$
\begin{array}{cc}
\psi_{\epsilon_{k}}^{(k)}(x) \propto & e^{-\int_{0}^{x} \alpha_{k}\left(y, \epsilon_{k}\right) d y} \\
\psi_{\epsilon_{k-1}}^{(k)}(x)= & \frac{A_{k}^{\dagger} \psi_{\epsilon_{k-1}}^{(k-1)}(x)}{\sqrt{\epsilon_{k-1}-\epsilon_{k}}} \\
\vdots \\
\psi_{\epsilon_{1}}^{(k)}(x)= & \frac{A_{k}^{\dagger} \ldots A_{2}^{\dagger} \psi_{\epsilon_{1}}^{(1)}(x)}{\sqrt{\left(\epsilon_{1}-\epsilon_{2}\right) \ldots\left(\epsilon_{1}-\epsilon_{k}\right)}} \\
\psi_{n}^{(k)}(x)= & \frac{A_{k}^{\dagger} \ldots A_{1}^{\dagger} \psi_{n}^{(0)}(x)}{\sqrt{\left(E_{n}-\epsilon_{1}\right) \ldots\left(E_{n}-\epsilon_{k}\right)}}
\end{array}
$$

The corresponding eigenvalues are $\left\{\epsilon_{i}, E_{n}, i=k, \ldots, 1, n=0,1, \ldots\right\}$.

In order to have the scheme complete, let us remember how are intertwined the $H_{i}$ 's:

$$
H_{i} A_{i}^{\dagger}=A_{i}^{\dagger} H_{i-1} \quad i=1, \ldots, k
$$

Thus, departing from $H_{0}$ we have generated a chain of factorized Hamiltonians:

$$
\begin{gathered}
H_{i}=A_{i}^{\dagger} A_{i}+\epsilon_{i}=A_{i+1} A_{i+1}^{\dagger}+\epsilon_{i+1} \quad i=1, \ldots, k-1 \\
H_{k}=A_{k}^{\dagger} A_{k}+\epsilon_{k}
\end{gathered}
$$

where the end potential $V_{k}(x)$ can be recursively determined by means of (2930). Thus, we require the mentioned $k$ solutions $\alpha_{1}\left(x, \epsilon_{i}\right), i=1, \ldots, k$ to the initial Riccati equation (6)), which means to have $k$ non-equivalent factorizations of the Hamiltonian $H_{0}$ :

$$
H_{0}=\frac{1}{2}\left(\frac{d}{d x}+\alpha_{1}\left(x, \epsilon_{i}\right)\right)\left(-\frac{d}{d x}+\alpha_{1}\left(x, \epsilon_{i}\right)\right)+\epsilon_{i} \quad i=1, \ldots, k
$$

Let us notice that there is a $k$-th order differential operator, $B_{k}^{\dagger}=A_{k}^{\dagger} \ldots A_{1}^{\dagger}$, intertwining the initial $H_{0}$ and final Hamiltonians $H_{k}$ :

$$
H_{k} B_{k}^{\dagger}=B_{k}^{\dagger} H_{0}
$$

From equations (31) we get:

$$
B_{k}^{\dagger} \psi_{n}^{(0)}=\sqrt{\left(E_{n}-\epsilon_{1}\right) \ldots\left(E_{n}-\epsilon_{k}\right)} \psi_{n}^{(k)}
$$

while from the adjoint to (36) it turns out that:

$$
B_{k} \psi_{n}^{(k)}=\sqrt{\left(E_{n}-\epsilon_{1}\right) \ldots\left(E_{n}-\epsilon_{k}\right)} \psi_{n}^{(0)}
$$

These equations lead to the higher-order supersymmetric quantum mechanics H-SUSY QM [59-69]. In this treatment the standard SUSY algebra with two generators [4]

$$
\left[Q_{i}, H_{\mathrm{ss}}\right]=0 \quad\left\{Q_{i}, Q_{j}\right\}=\delta_{i j} H_{\mathrm{ss}} \quad i, j=1,2
$$


is realized with the aid of $B_{k}$ and $B_{k}^{\dagger}$ :

$$
\begin{gathered}
Q=\left(\begin{array}{cc}
0 & 0 \\
B_{k} & 0
\end{array}\right) \quad Q^{\dagger}=\left(\begin{array}{cc}
0 & B_{k}^{\dagger} \\
0 & 0
\end{array}\right) \\
H_{\mathrm{ss}}=\left\{Q, Q^{\dagger}\right\}=\left(\begin{array}{cc}
B_{k}^{\dagger} B_{k} & 0 \\
0 & B_{k} B_{k}^{\dagger}
\end{array}\right)
\end{gathered}
$$

where $Q_{1}=\left(Q^{\dagger}+Q\right) / \sqrt{2}, Q_{2}=i\left(Q-Q^{\dagger}\right) / \sqrt{2}$. Due to

$$
\begin{aligned}
B_{k}^{\dagger} B_{k} & =A_{k}^{\dagger} \ldots A_{1}^{\dagger} A_{1} \ldots A_{k}=A_{k}^{\dagger} \ldots A_{2}^{\dagger}\left(H_{1}-\epsilon_{1}\right) A_{2} \ldots A_{k} \\
& =A_{k}^{\dagger} \ldots A_{3}^{\dagger}\left(H_{2}-\epsilon_{1}\right)\left(H_{2}-\epsilon_{2}\right) A_{3} \ldots A_{k}=\left(H_{k}-\epsilon_{1}\right) \ldots\left(H_{k}-\epsilon_{k}\right) \\
B_{k} B_{k}^{\dagger} & =A_{1} \ldots A_{k} A_{k}^{\dagger} \ldots A_{1}^{\dagger}=A_{1} \ldots A_{k-1}\left(H_{k-1}-\epsilon_{k}\right) A_{k-1}^{\dagger} \ldots A_{1}^{\dagger} \\
& =\left(H_{0}-\epsilon_{1}\right) \ldots\left(H_{0}-\epsilon_{k}\right)
\end{aligned}
$$

it turns out that the SUSY generator $H_{\mathrm{ss}}$ is a $k$-th order polynomial

$$
H_{\mathrm{ss}}=\left(H_{\mathrm{s}}^{p}-\epsilon_{1}\right) \ldots\left(H_{\mathrm{s}}^{p}-\epsilon_{k}\right)
$$

of the physical Hamiltonian $H_{\mathrm{s}}^{p}$ involving the intertwined Hamiltonians $H_{0}$ and $H_{k}$

$$
H_{\mathrm{s}}^{p}=\left(\begin{array}{cc}
H_{k} & 0 \\
0 & H_{0}
\end{array}\right)
$$

In particular, if $k=1$ we will get the standard SUSY QM for which $H_{\mathrm{ss}}=\left(H_{\mathrm{s}}^{p}-\epsilon_{1}\right)$, i.e., $H_{\mathrm{ss}}$ is linear in $H_{\mathrm{s}}^{p}$. If $k=2$ we will get the quadratic superalgebra, or SUSUSY QM due to $H_{\mathrm{ss}}=\left(H_{\mathrm{s}}^{p}-\epsilon_{1}\right)\left(H_{\mathrm{s}}^{p}-\epsilon_{2}\right)[59,62,63]$. 


\section{Higher-order supersymmetric quantum mechanics: direct approach}

In the direct procedure to the H-SUSY QM it is supposed from the very beginning that the intertwining operator in (36) is of $k$-th order:

$$
B_{k}^{\dagger}=2^{-k / 2}\left[(-1)^{k} \frac{d^{k}}{d x^{k}}+g_{k-1}(x) \frac{d^{k-1}}{d x^{k-1}}+\cdots+g_{1}(x) \frac{d}{d x}+g_{0}(x)\right]
$$

where the real functions $\left\{g_{i}(x), i=0, \ldots, k-1\right\}$ can in principle be determined through an approach similar to the one followed in the first-order case. Let us notice that the formulae (36, 43) are still valid in this case, but the procedure to derive them is long and involved. Here we present just the simplest case with $k=2$. This will illustrate clearly the advantages to design spectra supplied by the direct procedure compared with the iterative one.

\subsection{Second-order supersymmetric quantum mechanics}

The second-order supersymmetric quantum mechanics [59-62,77,96] arises when considering a second order intertwining operator such that

$$
\begin{aligned}
& H_{2} B_{2}^{\dagger}=B_{2}^{\dagger} H_{0} \\
& H_{i}=-\frac{1}{2} \frac{d^{2}}{d x^{2}}+V_{i}(x) \quad i=0,2 \\
& B_{2}^{\dagger}=\frac{1}{2}\left(\frac{d^{2}}{d x^{2}}-\eta(x) \frac{d}{d x}+\gamma(x)\right)
\end{aligned}
$$

The calculation of the left hand side of (45) leads to:

$$
\begin{aligned}
2 H_{2} B_{2}^{\dagger}= & -\frac{1}{2} \frac{d^{4}}{d x^{4}}+\frac{\eta}{2} \frac{d^{3}}{d x^{3}}+\left(\eta^{\prime}-\frac{\gamma}{2}+V_{2}\right) \frac{d^{2}}{d x^{2}} \\
& +\left(\frac{\eta^{\prime \prime}}{2}-\gamma^{\prime}-\eta V_{2}\right) \frac{d}{d x}+\gamma V_{2}-\frac{\gamma^{\prime \prime}}{2}
\end{aligned}
$$

The corresponding right hand side provides:

$$
\begin{aligned}
2 B_{2}^{\dagger} H_{0}= & -\frac{1}{2} \frac{d^{4}}{d x^{4}}+\frac{\eta}{2} \frac{d^{3}}{d x^{3}}+\left(V_{0}-\frac{\gamma}{2}\right) \frac{d^{2}}{d x^{2}} \\
& +\left(2 V_{0}^{\prime}-\eta V_{0}\right) \frac{d}{d x}+V_{0}^{\prime \prime}-\eta V_{0}^{\prime}+\gamma V_{0}
\end{aligned}
$$

The coefficients of the same powers of $d / d x$ in (46 47) must be equal, and thus we arrive at:

$$
\begin{gathered}
V_{2}=V_{0}-\eta^{\prime} \\
\frac{\eta^{\prime \prime}}{2}-\gamma^{\prime}-\eta V_{2}=2 V_{0}^{\prime}-\eta V_{0} \\
\gamma V_{2}-\frac{\gamma^{\prime \prime}}{2}=V_{0}^{\prime \prime}-\eta V_{0}^{\prime}+\gamma V_{0}
\end{gathered}
$$


By substituting (48) in (49) and solving for $\gamma^{\prime}$ we get:

$$
\gamma^{\prime}=\frac{\eta^{\prime \prime}}{2}+\eta \eta^{\prime}-2 V_{0}^{\prime}
$$

Integrating this equation with respect to $x$ :

$$
\gamma=\frac{\eta^{\prime}}{2}+\frac{\eta^{2}}{2}-2 V_{0}+d
$$

where $d$ is a real constant. By plugging (48) in (50) and solving for $\gamma^{\prime \prime}$ we have:

$$
\gamma^{\prime \prime}=2\left(\eta V_{0}^{\prime}-V_{0}^{\prime \prime}-\gamma \eta^{\prime}\right)
$$

Taking the derivative of (51) with respect to $x$ we get:

$$
\gamma^{\prime \prime}=\frac{\eta^{\prime \prime \prime}}{2}+\eta \eta^{\prime \prime}+\eta^{2}-2 V_{0}^{\prime \prime}
$$

If we substitute (52) and (54) in (53) we arrive at

$$
\frac{\eta^{\prime \prime \prime}}{2}+\eta \eta^{\prime \prime}+2 \eta^{\prime 2}=2\left(\eta V_{0}^{\prime}+2 \eta^{\prime} V_{0}\right)-\eta^{2} \eta^{\prime}-2 d \eta^{\prime}
$$

If we multiply the previous equation by $\eta$, then add and subtract $\eta^{\prime} \eta^{\prime \prime} / 2$ we arrive at:

$$
2 \frac{d}{d x}\left(\eta^{2} V_{0}\right)=\frac{\eta \eta^{\prime \prime \prime}}{2}+\frac{\eta^{\prime} \eta^{\prime \prime}}{2}+\frac{d}{d x}\left(\eta^{2} \eta^{\prime}\right)+\eta^{3} \eta^{\prime}+2 d \eta \eta^{\prime}-\frac{\eta^{\prime} \eta^{\prime \prime}}{2}
$$

Integrating with respect to $x$ and moving all the terms to the right hand side we get:

$$
\frac{\eta \eta^{\prime \prime}}{2}-\frac{\eta^{2}}{4}+\eta^{2} \eta^{\prime}+\frac{\eta^{4}}{4}-2 V_{0} \eta^{2}+d \eta^{2}+c=0
$$

being $c$ a real constant. Therefore, given $V_{0}(x)$ the new potential $V_{2}(x)$ and the function $\gamma(x)$ are obtained through (48) and (52) once we know explicitly a solution $\eta(x)$ of (55). To get it, we make the Ansätz [65,66,77]

$$
\eta^{\prime}=-\eta^{2}+2 \beta \eta+2 \xi
$$

where $\beta(x)$ and $\xi(x)$ are functions to be determined. Therefore:

$$
\begin{aligned}
\eta^{\prime \prime} & =-2 \eta \eta^{\prime}+2 \beta^{\prime} \eta+2 \beta \eta^{\prime}+2 \xi^{\prime} \\
\frac{\eta \eta^{\prime \prime}}{2} & =-\eta^{2} \eta^{\prime}+\beta^{\prime} \eta^{2}+\beta \eta \eta^{\prime}+\eta \xi^{\prime} \\
-\frac{\eta^{\prime 2}}{4} & =-\frac{\eta^{4}}{4}+\beta \eta^{3}+\left(\xi-\beta^{2}\right) \eta^{2}-2 \beta \xi \eta-\xi^{2}
\end{aligned}
$$

The substitution of (58) and (59) into (55) leads to:

$$
\begin{gathered}
\beta^{\prime} \eta^{2}+\beta \eta \eta^{\prime}+\eta \xi^{\prime}+\beta \eta^{3}+\left(\xi-\beta^{2}\right) \eta^{2} \\
-2 \beta \xi \eta-\xi^{2}-2 V_{0} \eta^{2}+d \eta^{2}+c=0
\end{gathered}
$$


and using again (56) to eliminate $\eta^{\prime}$ in the previous equation we arrive at:

$$
\left(\beta^{\prime}+\beta^{2}-2 V_{0}+\xi+d\right) \eta^{2}+\xi^{\prime} \eta-\xi^{2}+c=0
$$

Since (61) must be valid for arbitrary $\eta$, the coefficients of each power of $\eta$ must vanish, which leads to $\xi^{2} \equiv c$. Therefore:

$$
\beta^{\prime}(x)+\beta^{2}(x)=2\left[V_{0}(x)-\epsilon\right] \quad \epsilon=\frac{d+\xi}{2}
$$

Alternatively, we can work with the Schrödinger equation related to (62) through the change $\beta=u^{(0)^{\prime}} / u^{(0)}[97]$ :

$$
-\frac{1}{2} u^{(0)^{\prime \prime}}+V_{0} u^{(0)}=\epsilon u^{(0)}
$$

Depending on whether $c$ is zero or not, $\xi$ vanishes or takes two different values $\xi= \pm \sqrt{c}$. If $c=0$, we need to solve one equation of form (62) and then the resulting (56) for $\eta(x)$. If $c \neq 0$ there will be two different equations of type (62), with factorization energies $\epsilon_{1} \equiv(d+\sqrt{c}) / 2$ and $\epsilon_{2} \equiv(d-\sqrt{c}) / 2$. Once we solve them, it is possible to construct algebraically a common solution $\eta(x)$ of the corresponding pair of equations (56). There is an obvious difference between the real case with $c>0$ and the complex case with $c<0$; thus, it follows a natural scheme of classification for the solutions $\eta(x)$ based on the sign of $c$.

\subsection{Classification of the second-order SUSY transformations}

\subsubsection{The real case with $c>0$}

Here we have that $\epsilon_{1}, \epsilon_{2} \in \mathbb{R}, \epsilon_{1} \neq \epsilon_{2}$, and the corresponding Riccati solutions of (62) are denoted by $\beta_{1}(x), \beta_{2}(x)$ respectively. The associated pair of equations (56) become

$$
\begin{aligned}
& \eta^{\prime}(x)=-\eta^{2}(x)+2 \beta_{1}(x) \eta(x)+2\left(\epsilon_{1}-\epsilon_{2}\right) \\
& \eta^{\prime}(x)=-\eta^{2}(x)+2 \beta_{2}(x) \eta(x)+2\left(\epsilon_{2}-\epsilon_{1}\right)
\end{aligned}
$$

By subtracting them we get algebraically $\eta(x)$ in terms of $\epsilon_{1}, \epsilon_{2}$ and $\beta_{1}(x), \beta_{2}(x)$ :

$$
\eta(x)=-\frac{2\left(\epsilon_{1}-\epsilon_{2}\right)}{\beta_{1}(x)-\beta_{2}(x)}
$$

If the corresponding Schrödinger solutions are used we have

$$
\eta(x)=\frac{2\left(\epsilon_{1}-\epsilon_{2}\right) u_{1}^{(0)} u_{2}^{(0)}}{W\left(u_{1}^{(0)}, u_{2}^{(0)}\right)}=\frac{W^{\prime}\left(u_{1}^{(0)}, u_{2}^{(0)}\right)}{W\left(u_{1}^{(0)}, u_{2}^{(0)}\right)}
$$

where $W(f, g)=f g^{\prime}-g f^{\prime}$ denotes the Wronskian of $f$ and $g$. It follows that the second-order SUSY partner potentials $V_{2}(x)$ have no added singularities if $W\left(u_{1}^{(0)}, u_{2}^{(0)}\right)$ has no zeros.

The spectrum of $H_{2}, \operatorname{Sp}\left(H_{2}\right)$, will differ from $\operatorname{Sp}\left(H_{0}\right)$ depending on the normalizability of the two mathematical eigenfunctions $\psi_{\epsilon_{1}}^{(2)}, \psi_{\epsilon_{2}}^{(2)}$ of $H_{2}$ associated to $\epsilon_{1}$ and $\epsilon_{2}$ which belong to the kernel of $B$ :

$$
B \psi_{\epsilon_{j}}^{(2)}=0 \quad H_{2} \psi_{\epsilon_{j}}^{(2)}=\epsilon_{j} \psi_{\epsilon_{j}}^{(2)} \quad j=1,2
$$


For the solution associated to $\epsilon_{1}$ we explicitly have

$$
\begin{aligned}
& \psi_{\epsilon_{1}}^{(2)^{\prime \prime}}+\eta \psi_{\epsilon_{1}}^{(2)^{\prime}}+\left(\gamma+\eta^{\prime}\right) \psi_{\epsilon_{1}}^{(2)}=0 \\
& \psi_{\epsilon_{1}}^{(2)^{\prime \prime}}=2\left(V_{2}-\epsilon_{1}\right) \psi_{\epsilon_{1}}^{(2)}
\end{aligned}
$$

By substituting (67) in (66) one finds:

$$
\eta \psi_{\epsilon_{1}}^{(2)^{\prime}}+\left(\gamma+\eta^{\prime}+2 V_{2}-2 \epsilon_{1}\right) \psi_{\epsilon_{1}}^{(2)}=0
$$

and using the expressions for $V_{2}$ and $\gamma$ given in (48) and (52) with $d=\epsilon_{1}+\epsilon_{2}$ we get:

$$
\frac{\psi_{\epsilon_{1}}^{(2)^{\prime}}}{\psi_{\epsilon_{1}}^{(2)}}=\frac{\eta^{\prime} / 2-\eta^{2} / 2+\epsilon_{1}-\epsilon_{2}}{\eta}
$$

But from our Ansätz

$$
\eta^{2}=-\eta^{\prime}+2 \beta_{1} \eta+2\left(\epsilon_{1}-\epsilon_{2}\right)
$$

so that

$$
\frac{\psi_{\epsilon_{1}}^{(2)^{\prime}}}{\psi_{\epsilon_{1}}^{(2)}}=\frac{\eta^{\prime}}{\eta}-\beta_{1}=\frac{\eta^{\prime}}{\eta}-\frac{u_{1}^{(0)^{\prime}}}{u_{1}^{(0)}}
$$

Therefore

$$
\psi_{\epsilon_{1}}^{(2)} \propto \frac{\eta}{u_{1}^{(0)}} \propto \frac{u_{2}^{(0)}}{W\left(u_{1}^{(0)}, u_{2}^{(0)}\right)}
$$

A similar procedure leads to

$$
\psi_{\epsilon_{2}}^{(2)} \propto \frac{u_{1}^{(0)}}{W\left(u_{1}^{(0)}, u_{2}^{(0)}\right)}
$$

Concerning the possibilities for manipulating spectra offered by the second-order supersymmetric quantum mechanics, we have found a heuristic criterion providing some interesting information. Remember that

$$
B_{2} B_{2}^{\dagger}=\left(H_{0}-\epsilon_{1}\right)\left(H_{0}-\epsilon_{2}\right)
$$

which has to be positive definite on $\mathcal{H}$. In particular, this has to be valid on the basis of energy eigenstates $\left|\psi_{n}^{0}\right\rangle$ of $H_{0}$, and thus we have

$$
\left\langle\psi_{n}^{0}\left|B_{2} B_{2}^{\dagger}\right| \psi_{n}^{0}\right\rangle=\left(E_{n}-\epsilon_{1}\right)\left(E_{n}-\epsilon_{2}\right) \geq 0 \forall n
$$

This opens unexpected possibilities for the positions of the new levels $\epsilon_{1}, \epsilon_{2}$. A non exhaustive list of several interesting situations useful for the spectral design is presented next.

a) Our heuristic criterion indicates that if $\epsilon_{2}<\epsilon_{1}<E_{0}$ it is possible to find $u_{1}^{(0)}$ and $u_{2}^{(0)}$ such that $W\left(u_{1}^{(0)}, u_{2}^{(0)}\right)$ is nodeless and $\psi_{\epsilon_{1}}^{(2)}, \psi_{\epsilon_{2}}^{(2)}$ are normalizable. Indeed, with this ordering of $\epsilon_{1}$ and $\epsilon_{2}$ the right choice is to take $u_{1}^{(0)}$ nodeless and $u_{2}^{(0)}$ having one zero at $x_{0}$, i.e., $u_{2}^{(0)}\left(x_{0}\right)=0$. Since $W^{\prime}\left(u_{1}^{(0)}, u_{2}^{(0)}\right)=2\left(\epsilon_{1}-\epsilon_{2}\right) u_{1}^{(0)} u_{2}^{(0)}$, it turns out 


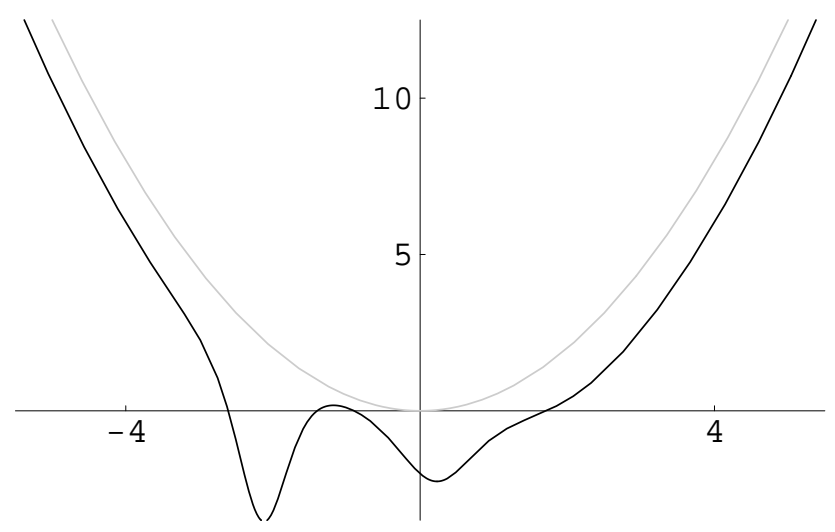

Figure 1: Second-order SUSY partner potential $V_{2}(x)$ (black curve) of the harmonic oscillator (gray curve) generated by using two Schrödinger solutions $u_{2}^{(0)}, u_{1}^{(0)}$ of the form (87) with $\epsilon_{2}=-1.2, \nu_{2}=1.1$ and $\epsilon_{1}=-1, \nu_{1}=0.9$ respectively. Here $\operatorname{Sp}\left(H_{2}\right)=\left\{-1.2,-1, \frac{1}{2}, \frac{3}{2}, \ldots\right\}$

that $W\left(u_{1}^{(0)}, u_{2}^{(0)}\right)$ has one critical point at $x_{0}$. Moreover, we have that at this point $W^{\prime \prime}\left(u_{1}^{(0)}, u_{2}^{(0)}\right) / W\left(u_{1}^{(0)}, u_{2}^{(0)}\right)=2\left(\epsilon_{1}-\epsilon_{2}\right)>0$ which implies that the Wronskian acquires either a minimum positive or a maximum negative value at $x_{0}$. Hence, $W\left(u_{1}^{(0)}, u_{2}^{(0)}\right)$ is nodeless. The spectrum of the new Hamiltonian is $\operatorname{Sp}\left(H_{2}\right)=\left\{\epsilon_{2}, \epsilon_{1}, E_{0}, E_{1}, \ldots\right\}$ (see a potential $V_{2}(x)$ with this kind of spectrum in figure 1). Notice that this case coincides with the one typically discussed when the second-order transformation is achieved through the iteration of two first-order transformations.

b) If we choose $E_{i}<\epsilon_{2}<\epsilon_{1}<E_{i+1}$, our heuristic criterion suggests that perhaps we can find $u_{1}^{(0)}$ and $u_{2}^{(0)}$ such that $W\left(u_{1}^{(0)}, u_{2}^{(0)}\right)$ is nodeless and $\psi_{\epsilon_{1}}^{(2)}, \psi_{\epsilon_{2}}^{(2)}$ are normalizable [96,98-100]. We would get then that $\operatorname{Sp}\left(H_{2}\right)=\left\{E_{0}, \ldots, E_{i}, \epsilon_{2}, \epsilon_{1}, E_{i+1}, \ldots\right\}$ (see figure 2 ). This possibility becomes true if we select $u_{2}^{(0)}$ and $u_{1}^{(0)}$ having $i+2$ and $i+1$ nodes respectively. Indeed, due to the oscillatory theorem, which can be translated as the fact that between two zeros of $u_{2}^{(0)}$ there is at least one zero of $u_{1}^{(0)}$, it turns out that these $2 i+3$ nodes are alternating. These zeros, ordered as $x_{0}<x_{1}<\cdots<x_{2 i+2}$, are as well the critical points of $W\left(u_{1}^{(0)}, u_{2}^{(0)}\right) \equiv W(x)$. Since $W\left(x_{j}\right) / W\left(x_{j+1}\right)>0$, then $W(x)$ conserves its sign (i.e., it is nodeless) in the interval $\left(x_{j}, x_{j+1}\right)$. Therefore, it does not have zeros in the domain $\left(x_{0}, x_{2 i+2}\right)$. Finally, since $x_{0}$ is a node of $u_{2}^{(0)}$, then $W^{\prime \prime}\left(x_{0}\right) / W\left(x_{0}\right)=2\left(\epsilon_{1}-\epsilon_{2}\right)>0$. Hence, $W(x)$ acquires either a maximum negative or a minimum positive value at $x_{0}$. In both cases $W(x)$ never crosses the $x$-axis in the interval $\left(-\infty, x_{0}\right)$. A similar treatment leads us to conclude that the Wronskian does not vanish in $\left(x_{2 i+2}, \infty\right)$, and therefore it is nodeless in the full real line.

c) If $\epsilon_{2}=E_{i}, \epsilon_{1}=E_{i+1}$, and $u_{2}^{(0)}=\psi_{i}^{(0)}, u_{1}^{(0)}=\psi_{i+1}^{(0)}$, then $W\left(u_{1}^{(0)}, u_{2}^{(0)}\right)$ is nodeless but $\psi_{\epsilon_{1}}^{(2)}, \psi_{\epsilon_{2}}^{(2)}$ are non-normalizable. In order to prove this, let us notice that $u_{2}^{(0)}$ and $u_{1}^{(0)}$ have now $i$ and $i+1$ nodes respectively. Due to the null asymptotic behavior at $x \rightarrow \pm \infty$ of both $u_{1}^{(0)}, u_{2}^{(0)}$, it turns out that these $2 i+1$ zeros, ordered as $x_{0}<x_{1}<$ $\cdots<x_{2 i}$, are once again alternating, with $x_{0}$ and $x_{2 i}$ being now zeros of $u_{1}^{(0)}$. Using a 


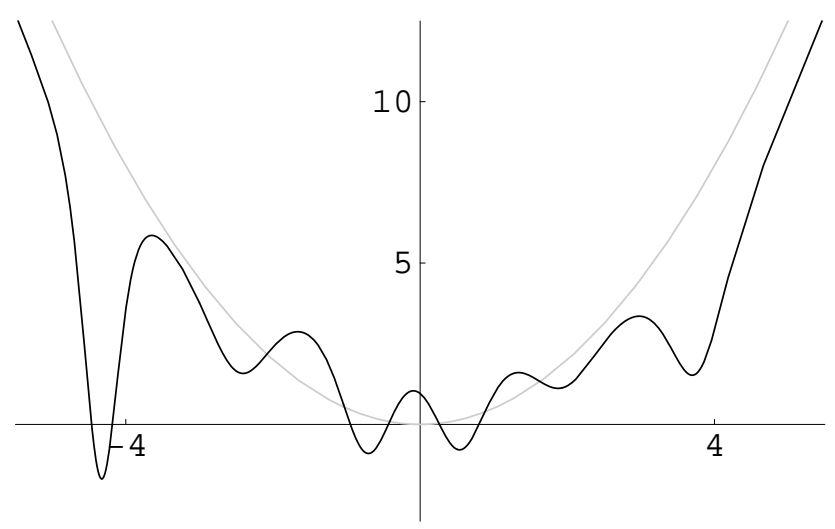

Figure 2: Second-order SUSY partner potential $V_{2}(x)$ (black curve) of the oscillator (gray curve) generated through two solutions $u_{2}^{(0)}, u_{1}^{(0)}$ of the form (87) with $\epsilon_{2}=3, \nu_{2}=0.9$ and $\epsilon_{1}=3.2, \nu_{1}=1.1$ respectively. We have that $\operatorname{Sp}\left(H_{2}\right)=\left\{\frac{1}{2}, \frac{3}{2}, \frac{5}{2}, 3,3.2, \frac{7}{2}, \ldots\right\}$.

reasoning similar to that used in the previous case, $W(x)$ becomes nodeless in $\left(x_{0}, x_{2 i}\right)$. On the other hand, $W(x)$ is monotonic in the interval $\left(-\infty, x_{0}\right)$ and $W^{\prime \prime}\left(x_{0}\right) / W\left(x_{0}\right)=$ $2\left(\epsilon_{2}-\epsilon_{1}\right)<0$, which implies that $W(x)$ has either a maximum positive or a minimum negative value at $x=x_{0}$. Since $\lim _{x \rightarrow-\infty} W(x)=0$, it turns out that the only node which $W(x)$ has in the interval $\left(-\infty, x_{0}\right)$ is an asymptotic zero as $x \rightarrow-\infty$. A similar procedure shows that in $\left(x_{2 i}, \infty\right) W(x)$ has an asymptotic zero as $x \rightarrow \infty$. In conclusion, the Wronskian is nodeless in the full real line, except by asymptotic null behaviors at $x \rightarrow \pm \infty$. This implies that the second-order SUSY transformation is non-singular inside the initial domain of definition. As the intertwining operator respect the boundary conditions of the eigenfunctions of $H_{0}$ (except that now the eigenfunctions of $H_{2}$ associated to $E_{i}$ and $E_{i+1}$ are not square-integrable anymore), it turns out that $\operatorname{Sp}\left(H_{2}\right)=\left\{E_{0}, \ldots, E_{i-1}, E_{i+2}, \ldots\right\}$, i.e., somehow we have 'deleted' the two levels $E_{i}, E_{i+1}$ in order to generate $V_{2}(x)$.

According to the standard SUSY treatment, for which the new levels are always below the ground state energy of the initial Hamiltonian, the previous cases (b) and (c) are somehow unexpected, supplying us with more freedom for manipulating spectra. In principle these non-typical cases can be achieved through first-order SUSY transformations, but the corresponding interpretation is strange: in the first step we generate a singular potential $V_{1}$, with the corresponding singularities induced by the zeros of the transformation function which is employed. The second transformation removes then the singularities introduced in the first step to arrive at a final non-singular potential $V_{2}$. Next we will explore another interesting cases having unexpected positions for the new levels.

\subsubsection{The confluent case with $c=0$}

In this case $\xi=0$, therefore $\epsilon \equiv \epsilon_{1}=\epsilon_{2} \in \mathbb{R}$. Once we have found a Riccati solution $\beta(x)$ to (62) for the given $\epsilon$, we must solve the Bernoulli equation resulting from (56) $[69,101]$

$$
\eta^{\prime}=-\eta^{2}+2 \beta \eta
$$


In order to solve it, let us take $\eta=1 / y$. Then

$$
y^{\prime}+2 \beta y=1
$$

Hence

$$
y=\left[w_{0}+\int e^{2 \int \beta(x) d x} d x\right] e^{-2 \int \beta(x) d x}
$$

$w_{0}$ being a real constant. Thus, the general $\eta$-solution is given by

$$
\eta(x)=\frac{e^{2 \int \beta(x) d x}}{w_{0}+\int e^{2 \int \beta(x) d x} d x}
$$

In terms of the Schrödinger solution $u^{(0)}(x) \propto \exp \left[\int \beta(x) d x\right]$ we have

$$
\eta(x)=\frac{\left[u^{(0)}(x)\right]^{2}}{w_{0}+\int_{x_{0}}^{x}\left[u^{(0)}(y)\right]^{2} d y}=\frac{w^{\prime}(x)}{w(x)}
$$

where $x_{0}$ is a fixed point in the domain of $V_{0}(x)$ and, up to an unimportant constant factor:

$$
w(x)=w_{0}+\int_{x_{0}}^{x}\left[u^{(0)}(y)\right]^{2} d y
$$

In order that $V_{2}(x)$ will not have singularities, $w(x)$ must be nodeless. Since $w(x)$ is a nondecreasing monotonic function, a simple choice [101] (see also [95]) is to use Schrödinger solutions such that

$$
\lim _{x \rightarrow \infty} u^{(0)}(x)=0 \text { and } I_{+}=\int_{x_{0}}^{\infty}\left[u^{(0)}(y)\right]^{2} d y<\infty
$$

or

$$
\lim _{x \rightarrow-\infty} u^{(0)}(x)=0 \text { and } I_{-}=\int_{-\infty}^{x_{0}}\left[u^{(0)}(y)\right]^{2} d y<\infty
$$

In both cases it is possible to find a $w_{0}$-domain where $w(x)$ is nodeless [101]. For instance, if (74) is valid and $u^{(0)}(x)$ is a non-physical eigenfunction of $H_{0}$ associated to $\epsilon$, it turns out that $\lim _{x \rightarrow-\infty} w(x)=-\infty$ and $\lim _{x \rightarrow \infty} w(x)=w_{0}+I_{+}$. Thus, the domain for which $w(x)$ is nodeless is $w_{0} \leq-I_{+}$. A similar procedure implies that for non-physical transformation functions satisfying (75) the nodeless $w_{0}$-domain is $w_{0} \geq I_{-}$.

Similarly as in the case with $c>0$, it can be found now a function $\psi_{\epsilon}^{(2)}$ in the kernel of $B$ which is simultaneously an eigenfunction of $H_{2}$ with eigenvalue $\epsilon$. Indeed, let us notice that equations (66, [71) remain valid in this case, we just have to substitute $\epsilon_{1}$ and $\epsilon_{2}$ by $\epsilon$, $u_{1}^{(0)}$ by $u^{(0)}$ and $\beta_{1}$ by $\beta$. Thus:

$$
\psi_{\epsilon}^{(2)}(x) \propto \frac{\eta(x)}{u^{(0)}(x)}=\frac{u^{(0)}(x)}{w(x)}
$$

The spectrum of $H_{2}$ depends on the normalizability of $\psi_{\epsilon}^{(2)}$. In particular, for $\epsilon \geq E_{0}$ it is possible to find solutions $u^{(0)}$ satisfying (74) or (75) and such that $\psi_{\epsilon}^{(2)}$ is normalizable. 


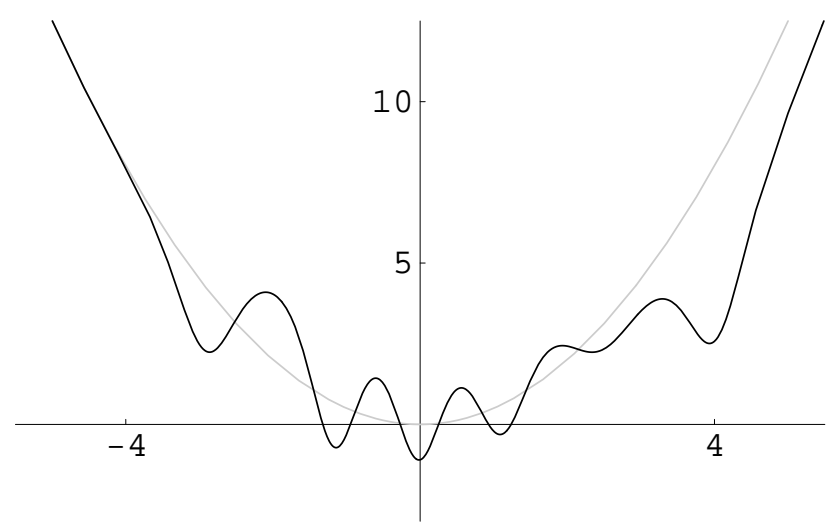

Figure 3: Confluent second-order SUSY partner potential $V_{2}(x)$ (black curve) of the oscillator (gray curve) generated by using a solution $u^{(0)}$ of the form (87) with $\epsilon=4, \nu=1$ and taking $w_{0}=5, x_{0}=0$ in (73). Now $\operatorname{Sp}\left(H_{2}\right)=\left\{\frac{1}{2}, \frac{3}{2}, \frac{5}{2}, \frac{7}{2}, 4, \frac{9}{2}, \ldots\right\}$.

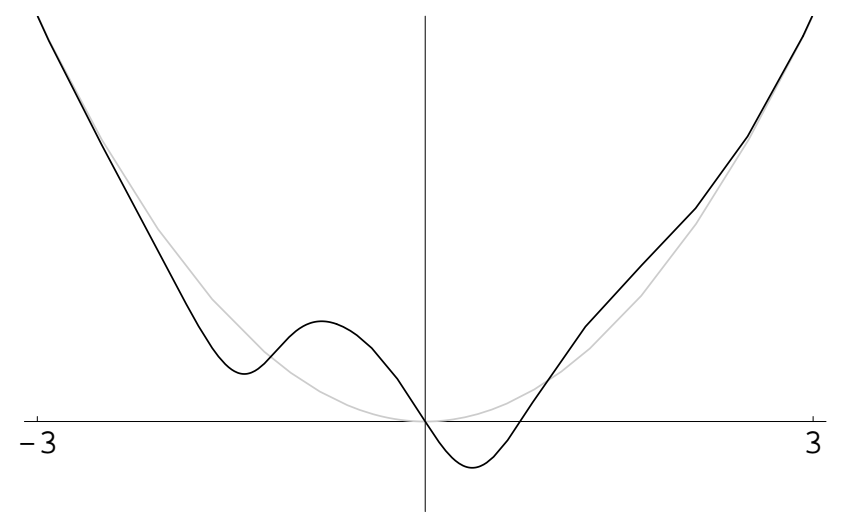

Figure 4: Confluent isospectral second-order SUSY partner potential $V_{2}(x)$ (black curve) of the oscillator (gray curve) generated by using the first excited state $u^{(0)}=\psi_{1}^{(0)}$ of the oscillator, with $\epsilon=E_{1}=\frac{3}{2}, w_{0}=1$ and $x_{0}=0$.

This means that the confluent second-order SUSY transformations allow the embedding of single energy levels above the ground state energy of $H_{0}$ (see figure 3 ). In addition, the physical solutions associated to the excited state levels of $H_{0}$ can be used as well as transformation functions, no matter the number of zeros they have in the domain of $V_{0}(x)$ (see figure 4). These two features cannot be achieved by iterating the first-order SUSY transformations without paying the price of introducing singularities at the intermediate potentials. As expected, these atypical spectral possibilities are consistent with our heuristic criterion previously formulated.

\subsubsection{The complex case with $c<0$}

If $c<0$ we have that $\epsilon_{1} \in \mathbb{C}$ and $\epsilon_{2}=\bar{\epsilon}_{1}$. Notice that our heuristic criterion allows this possibility without violating the positive nature of the operator $B_{2} B_{2}^{\dagger}$. We are going to analize just the case for which $V_{2}(x)$ is real valued, implying that $\beta_{2}(x)=\bar{\beta}_{1}(x)$. Following 


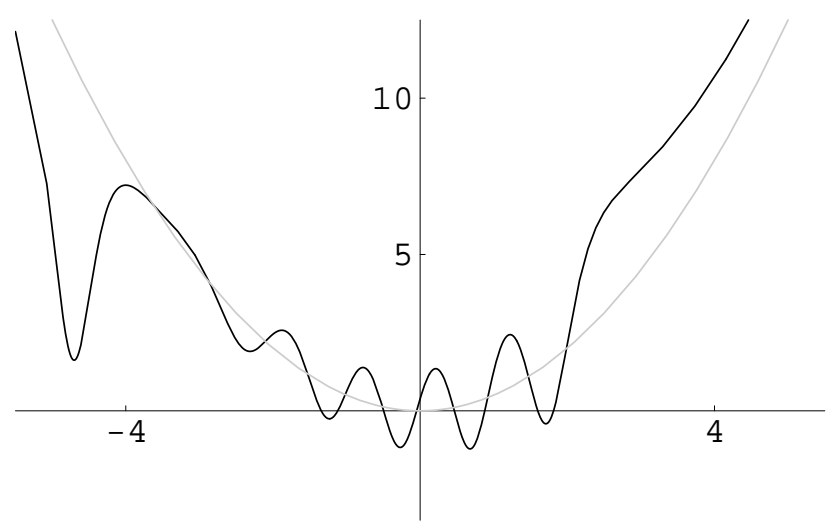

Figure 5: Real isospectral second-order SUSY partner potential $V_{2}(x)$ (black curve) of the oscillator (gray curve) generated by using a solution $u_{1}^{(0)}$ of the form (87) with $\nu_{1}=-1$ and $\epsilon_{1}=5.4+i / 20 \in \mathbb{C}$.

analogous steps as for the real case, one arrives to the solution $\eta(x)$ of (55) in terms of the (complex) solution $\beta_{1}(x)$ of the Riccati equation (62) associated to $\epsilon_{1}$ [102] (for the case when $V_{2}(x)$ is allowed to be complex see $\left.[103,104]\right)$ :

$$
\eta(x)=-\frac{2 \operatorname{Im}\left(\epsilon_{1}\right)}{\operatorname{Im}\left[\beta_{1}(x)\right]}
$$

Using the corresponding complex Schrödinger solution $u_{1}^{(0)}(x)$ we can write

$$
\eta(x)=\frac{w^{\prime}(x)}{w(x)} \quad w(x)=\frac{W\left(u_{1}^{(0)}, \bar{u}_{1}^{(0)}\right)}{2\left(\epsilon_{1}-\bar{\epsilon}_{1}\right)}
$$

In order to avoid the creation of singularities in $V_{2}(x), w(x)$ must be nodeless. Since $w^{\prime}(x)=\left|u_{1}^{(0)}(x)\right|^{2}$, it turns out that $w(x)$ is a non-decreasing monotonic function. Thus, to ensure that $w(x) \neq 0 \forall x \in \mathbb{R}$ it is sufficient that [102]

$$
\lim _{x \rightarrow \infty} u_{1}^{(0)}(x)=0 \quad \text { or } \quad \lim _{x \rightarrow-\infty} u_{1}^{(0)}(x)=0
$$

For transformation functions obeying the condition (177) it turns out that $V_{2}(x)$ is a real potential isospectral to $V_{0}(x)$ (see figure 5). Let us notice that, as in the previous cases, this transformation could be achieved by iterating the first-order SUSY, but the intermediate potentials would be complex. This is, perhaps, the reason why this case was almost unexplored in the past. From our viewpoint, however, it represents a very promising line of research for the next years (see e.g. [105]). 


\section{An example: the harmonic oscillator}

We are going to apply the previous techniques to the harmonic oscillator. Before doing that, however, it is convenient to discuss some generalities of a subset of nonlinear deformations of the Heisenberg algebra. We will realize later on the importance of such structures for the SUSY partners of the oscillator.

\subsection{Polynomial deformations of the Heisenberg algebra}

First of all let us remember the standard oscillator algebra

$$
\begin{gathered}
{\left[H, a^{\dagger}\right]=a^{\dagger} \quad[H, a]=-a} \\
{\left[a, a^{\dagger}\right]=1}
\end{gathered}
$$

for which the number operator is a linear function of $H$ :

$$
N=a^{\dagger} a=H-\frac{1}{2}
$$

On the other hand, the polynomial Heisenberg algebras of $m$-th order are deformations of the previous structure, where there are two standard commutation relationships

$$
\left[H, L^{ \pm}\right]= \pm L^{ \pm}
$$

and an atypical one characterizing the deformation:

$$
\left[L^{-}, L^{+}\right] \equiv N(H+1)-N(H)=P_{m}(H)
$$

where a generalization of the standard number operator is given by $N(H) \equiv L^{+} L^{-}$. The corresponding systems are described by Schrödinger Hamiltonians

$$
H=-\frac{1}{2} \frac{d^{2}}{d x^{2}}+V(x)
$$

being $L^{ \pm}$differential ladder operators of order $(m+1)$-th, $N(H)$ a polynomial of order $(m+1)$-th in $H$ factorized as

$$
N(H)=\prod_{i=1}^{m+1}\left(H-\mathcal{E}_{i}\right)
$$

and $P_{m}(H)$ a $m$-th order polynomial in $H$. The algebra (78) generated by $\left\{H, L^{-}, L^{+}\right\}$ provides information on the spectrum $\operatorname{Sp}(H)$ of $H[67,79,106,107]$. Indeed, let us consider the solution space of the $(m+1)$-th order differential equation (the kernel $K_{L^{-}}$of $\left.L^{-}\right)$:

$$
L^{-} \psi=0 \quad \Rightarrow \quad L^{+} L^{-} \psi=\prod_{i=1}^{m+1}\left(H-\mathcal{E}_{i}\right) \psi=0
$$

Notice that $K_{L^{-}}$is invariant under $H$ :

$$
L^{-} H \psi=(H+1) L^{-} \psi=0 \Rightarrow H \psi \in K_{L^{-}} \forall \psi \in K_{L^{-}}
$$




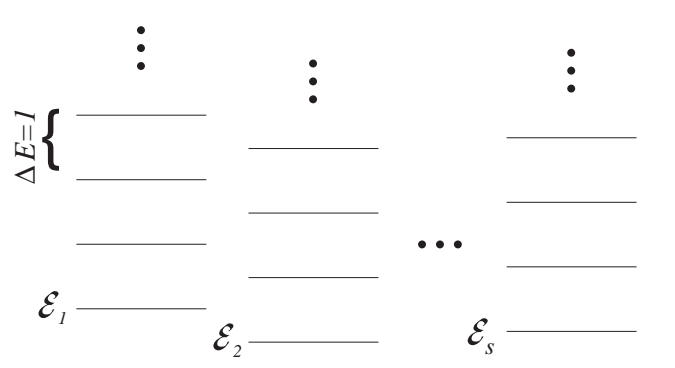

(a)

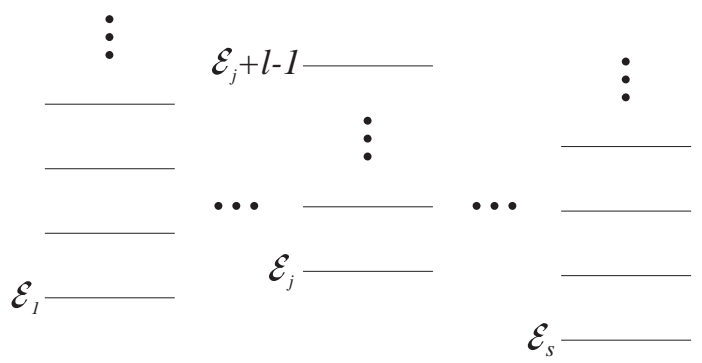

(b)

Figure 6: Possible spectra for the Hamiltonians satisfying (178) -(81) with $s$ physical extremal states. In case (a) $s$ infinite ladders are obtained by acting with $L^{+}$. In case (b) there are $s-1$ infinite ladders, and a finite one (the $j$-th one) is built out of $\psi_{\mathcal{E}_{j}}$ taking into account (84).

Thus, it is natural to select as the basis of $K_{L^{-}}$the functions which are simultaneously eigenstates of $H$ with eigenvalues $\mathcal{E}_{i}$

$$
H \psi_{\mathcal{E}_{i}}=\mathcal{E}_{i} \psi_{\mathcal{E}_{i}}
$$

These represent the extremal states for the $m+1$ mathematical ladders of spacing $\Delta E=1$ starting from $\mathcal{E}_{i}$. If $s$ of these states are physically meaningful, $\left\{\psi_{\mathcal{E}_{i}}, i=1, \ldots, s\right\}$, then by acting iteratively with $L^{+}$onto them $s$ physical energy ladders can be constructed (see figure 6 -a).

It could happen [67] that for the ladder starting from $\mathcal{E}_{j}$ there is a $l \in \mathbb{N}$ such that:

$$
\left(L^{+}\right)^{l-1} \psi_{\mathcal{E}_{j}} \neq 0 \quad\left(L^{+}\right)^{l} \psi_{\mathcal{E}_{j}}=0
$$

Then

$$
\begin{aligned}
& L^{-}\left(L^{+}\right)^{l} \psi_{\mathcal{E}_{j}}=L^{-} L^{+}\left(L^{+}\right)^{l-1} \psi_{\mathcal{E}_{j}} \\
& =\left(\prod_{i=1}^{m+1}\left(H+1-\mathcal{E}_{i}\right)\right)\left(L^{+}\right)^{l-1} \psi_{\mathcal{E}_{j}} \\
& =\left(\prod_{i=1}^{m+1}\left(\mathcal{E}_{j}+l-\mathcal{E}_{i}\right)\right)\left(L^{+}\right)^{l-1} \psi_{\mathcal{E}_{j}}=0
\end{aligned}
$$

This implies that another root of (81) must have the form $\mathcal{E}_{k}=\mathcal{E}_{j}+l, k \in\{s+1, \ldots, m+$ $1\}, j \in\{1, \ldots, s\}$. Hence, $\operatorname{Sp}(H)$ will contain $s-1$ infinite ladders and a finite one of length $l$, starting from $\mathcal{E}_{j}$ and ending at $\mathcal{E}_{j}+l-1$ (see figure 6-b).

We conclude that the spectrum of systems described by polynomial Heisenberg algebras of order $m$ can have at most $m+1$ infinite ladders. Notice that pairs of ladder operators for the harmonic oscillator satisfying (778] $L^{-}=a P(H), L^{+}=P(H) a^{\dagger}$, where $a, a^{\dagger}$ are the annihilation and creation operators of the oscillator and $P(H)$ is a real polynomial in $H$ [108]. These deformations are reducible since for the same system we already have ladder operators $a, a^{\dagger}$ obeying a much simpler algebra. Here we will be mainly interested in the search of irreducible deformed algebras. 


\subsection{SUSY partners of the oscillator}

Let us consider the harmonic oscillator potential

$$
V_{0}(x)=\frac{x^{2}}{2}
$$

The corresponding Hamiltonian has an equidistant spectrum with eigenfunctions and eigenvalues given by:

$$
\psi_{n}^{(0)}(x)=\sqrt{\frac{1}{2^{n} n ! \sqrt{\pi}}} H_{n}(x) e^{-\frac{x^{2}}{2}} \quad E_{n}=n+\frac{1}{2}, n=0,1, \ldots
$$

where $H_{n}(x)$ are the Hermite polynomials. In order to implement the several SUSY transformations discussed previously, we will solve the Schrödinger equation (63) for an arbitrary factorization energy $\epsilon$, namely:

$$
-\frac{1}{2} u^{(0)^{\prime \prime}}+\frac{x^{2}}{2} u^{(0)}=\epsilon u^{(0)}
$$

Suppose that $u^{(0)}=e^{-\frac{x^{2}}{2}} h(x)$. Therefore:

$$
h^{\prime \prime}-2 x h^{\prime}+(2 \epsilon-1) h=0
$$

By changing variables $y=x^{2}$ we arrive at:

$$
y \frac{d^{2} h}{d y^{2}}+(b-y) \frac{d h}{d y}-a h=0
$$

which is the confluent hypergeometric equation with $a=(1-2 \epsilon) / 4$ and $b=1 / 2$. The general solution of (86) leads to the general solution $u^{(0)}$ we were looking for:

$$
u^{(0)}(x)=e^{-\frac{x^{2}}{2}}\left[{ }_{1} F_{1}\left(a, \frac{1}{2} ; x^{2}\right)+2 \nu x \frac{\Gamma\left(a+\frac{1}{2}\right)}{\Gamma(a)}{ }_{1} F_{1}\left(a+\frac{1}{2}, \frac{3}{2} ; x^{2}\right)\right]
$$

where $\epsilon \in \mathbb{C}$ and ${ }_{1} F_{1}(a, b ; y)$ is the confluent hypergeometric (Kummer) function:

$$
{ }_{1} F_{1}(a, b ; y)=\frac{\Gamma(b)}{\Gamma(a)} \sum_{n=0}^{\infty} \frac{\Gamma(a+n)}{\Gamma(b+n)} \frac{y^{n}}{n !}
$$

Notice that, for $\epsilon<1 / 2$ and $|\nu| \leq 1$ the solution $u^{(0)}$ given in (87) is nodeless.

The explicit expressions for the SUSY partners of the oscillator can be calculated using (87) and, in general, they are too involved to be shown here. However, we will present the simplest derivation of a family of potentials isospectral to the oscillator [6], which originally was derived by Abraham and Moses through the Gelfand-Levitan formalism [95]. 


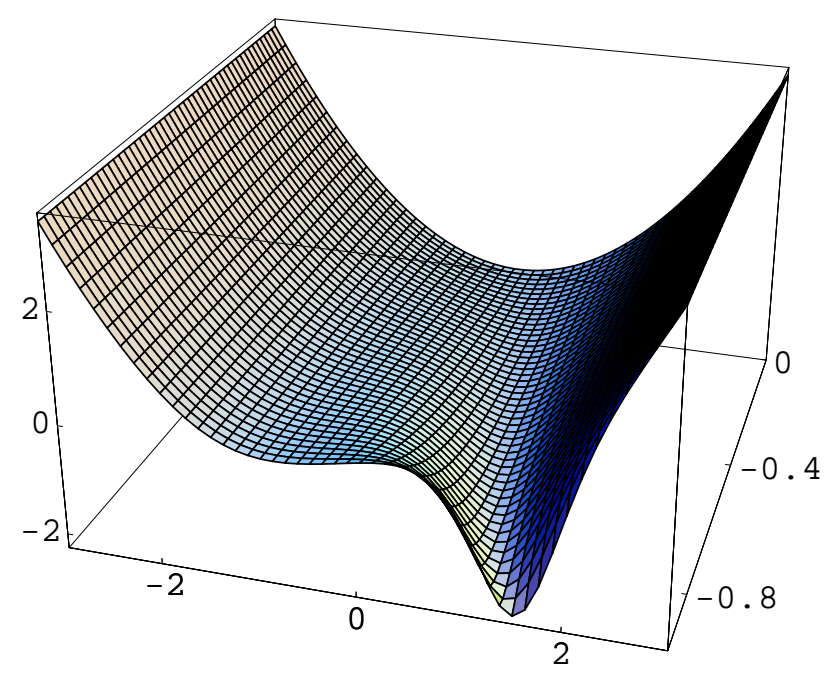

Figure 7: The Abraham-Moses family of potentials (89) as a function of $x$ and continuous values of the $\nu$-parameter in the domain $[-0.95,0]$.

\subsubsection{The Abraham-Moses potentials}

Let us make a first-order SUSY transformation employing the general Schrödinger solution (87) associated to $\epsilon=-1 / 2$, namely:

$$
u^{(0)}(x)=e^{\frac{x^{2}}{2}}[1+\nu \operatorname{Erf}(x)]
$$

where

$$
\operatorname{Erf}(x)=\frac{2}{\sqrt{\pi}} \int_{0}^{x} e^{-t^{2}} d t
$$

is the well known error function. The corresponding superpotential is given by

$$
\alpha_{1}(x, \epsilon)=x+\frac{2 \nu e^{-\frac{x^{2}}{2}}}{\sqrt{\pi}[1+\nu \operatorname{Erf}(x)]}
$$

which is precisely the one obtained by Mielnik through his generalized factorization [6]. The first-order SUSY partner potentials are given by:

$$
V_{1}(x)=\frac{x^{2}}{2}-\left(\frac{2 \nu e^{-\frac{x^{2}}{2}}}{\sqrt{\pi}[1+\nu \operatorname{Erf}(x)]}\right)^{\prime}-1
$$

Up to a displacement in the energy origin, this is the Abraham-Moses family of potentials isospectral to the oscillator [95]. A plot of these potentials in terms of the variable $x$ for continuous values of the parameter $\nu$ in $[-0.95,0]$ is given in figure 7 . Notice that for $\nu=0$ we recover the original oscillator potential. 
Let us perform now a confluent second-order SUSY transformation employing the ground state eigenfunction, namely:

$$
u^{(0)}=\frac{e^{-\frac{x^{2}}{2}}}{\pi^{1 / 4}} \quad \epsilon=\frac{1}{2}
$$

For the confluent algorithm the key $w$-function becomes (see equation (173)):

$$
w(x)=w_{0}+\frac{1}{2} \operatorname{Erf}(x)
$$

where we have made $x_{0}=0$. Taking now $1 / w_{0}=2 \nu$, the new potentials read:

$$
V_{2}(x)=\frac{x^{2}}{2}-\left(\frac{w^{\prime}}{w}\right)^{\prime}=\frac{x^{2}}{2}-\left(\frac{2 \nu e^{-\frac{x^{2}}{2}}}{\sqrt{\pi}[1+\nu \operatorname{Erf}(x)]}\right)^{\prime}
$$

These are precisely the already mentioned Abraham-Moses potentials (compare with (89)). Indeed, the procedure of derivation based in the confluent algorithm employing (90) essentially coincides with the Abraham-Moses treatment [95]. Although in this case the first-order SUSY and the confluent second-order SUSY algorithms led to the same family of potentials, this was a fortunate coincidence due to the huge symmetry of the oscillator. In the general case, the potentials derived through the first-order SUSY will not coincide with those generated through second-order SUSY transformations.

\subsection{Non-linear algebra of $H_{k}$}

Suppose now that we have applied a $k$-th order SUSY transformation to $V_{0}=x^{2} / 2$, producing then the potential $V_{k}$ by creating $k$ new energy levels. The spectrum of the end Hamiltonian $H_{k}$, intertwined with the harmonic oscillator through $B_{k}^{\dagger}$, will be $\left\{\epsilon_{i}, E_{n}=n+1 / 2, i=\right.$ $k, \ldots, 1, n=0,1, \ldots\}$, i.e., it contains a part isospectral to the oscillator plus $k$ additional levels $\epsilon_{i}, i=k, \ldots, 1$ placed by simplicity below $E_{0}=1 / 2$. Hence, it is possible that polynomial Heisenberg algebras rule the H-SUSY partners of the oscillator.

To analyze the algebraic structure characteristic of the Hamiltonians $H_{k}$, let us look for ladder operators which connect the eigenstates associated to the levels $E_{n}$. There is a natural construction for a pair of these operators $[6,67,84,85]$, which is guessed from equation (36), its adjoint and the standard intertwining relationship involving the oscillator Hamiltonian $H_{0}$ and its creation and annihilation operators $a^{\dagger}, a$ :

$$
\left(H_{0}-1\right) a^{\dagger}=a^{\dagger} H_{0} \quad\left(H_{0}+1\right) a=a H_{0}
$$

The construction is composed of three stages (see figure 8): i) first we 'move' the eigenvectors $\left|\psi_{n}^{k}\right\rangle$ of $H_{k}$, represented previously by the wave functions $\psi_{n}^{(k)}$, to the eigenvectors $\left|\psi_{n}^{0}\right\rangle$ of the oscillator Hamiltonian $H_{0}$ through the intertwining operator $B_{k}$. ii) Then, we move up $\left(\left|\psi_{n+1}^{0}\right\rangle\right)$ or down $\left(\left|\psi_{n-1}^{0}\right\rangle\right)$ on the ladder of $H_{0}$ by using $a^{\dagger}$ or $a$ respectively, which will cause the effective 'motion' up or down on the ladder of $H_{k}$. iii) Finally, we get back to the ladder of $H_{k}$ by acting $B_{k}^{\dagger}$ on $\left|\psi_{n+1}^{0}\right\rangle$ or $\left|\psi_{n-1}^{0}\right\rangle$. Thus, the 'natural' ladder operators for $H_{k}$ are:

$$
L_{k}=B_{k}^{\dagger} a B_{k} \quad L_{k}^{\dagger}=B_{k}^{\dagger} a^{\dagger} B_{k} \quad k=0,1, \ldots
$$




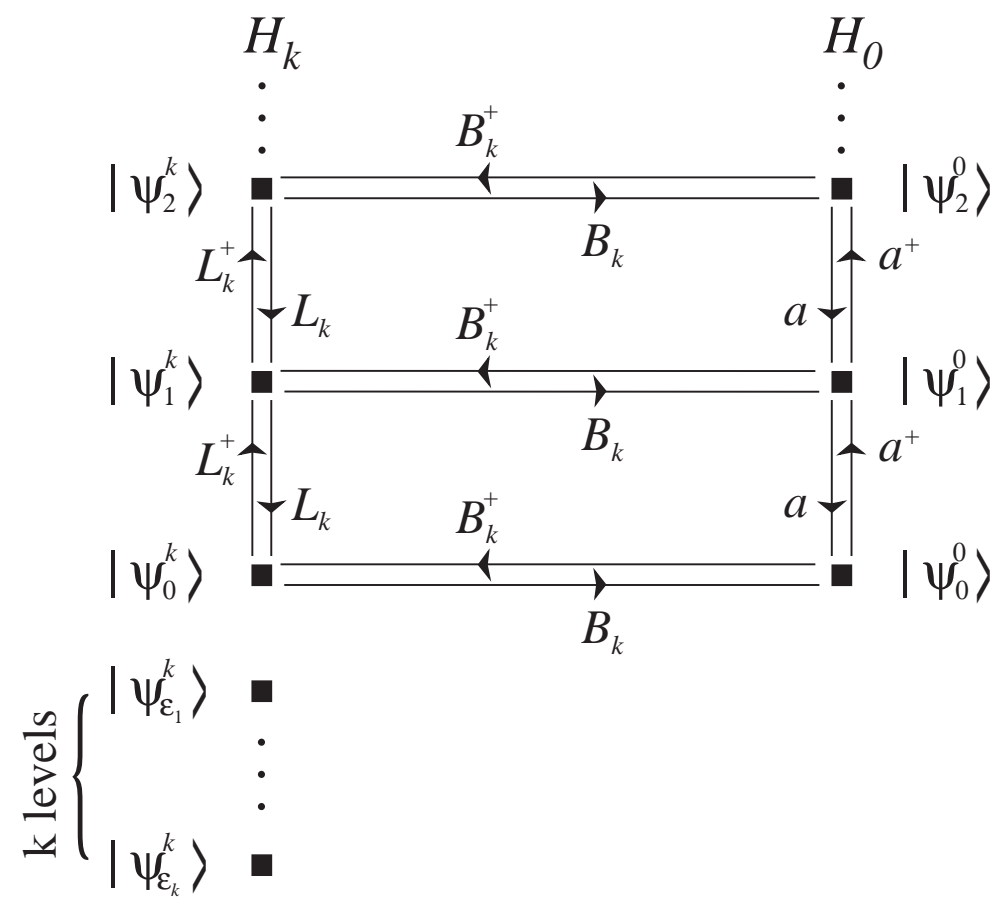

Figure 8: Diagram representing the action of the $k$-th order intertwining operators $B_{k}, B_{k}^{\dagger}$ and of the ladder operators $a, a^{\dagger}, L_{k}, L_{k}^{\dagger}$ for the Hamiltonians $H_{0}$ and $H_{k}$

For completeness, we have extended the intertwining relationship (36) to the case $k=0$ by assuming that $B_{0}^{\dagger}=B_{0}=I, I$ is the identity operator. The action of $L_{k}$ and $L_{k}^{\dagger}$ is drawn just onto the spectral points $E_{n}=n+1 / 2, n=0,1, \ldots$ because the eigenstates $\left\{\left|\psi_{\epsilon_{i}}^{k}\right\rangle, i=1, \ldots, k\right\}$ are annihilated by both $L_{k}$ and $L_{k}^{\dagger}$ since they are annihilated by $B_{k}$.

Notice that

$$
\begin{aligned}
H_{k} L_{k} & =H_{k} B_{k}^{\dagger} a B_{k}=B_{k}^{\dagger} H_{0} a B_{k}=B_{k}^{\dagger} a\left(H_{0}-1\right) B_{k} \\
& =B_{k}^{\dagger} a B_{k}\left(H_{k}-1\right)=L_{k}\left(H_{k}-1\right)
\end{aligned}
$$

i.e., $L_{k}$ and $L_{k}^{\dagger}$ are differential ladder operators of order $(2 k+1)$-th satisfying:

$$
\left[H_{k}, L_{k}\right]=-L_{k} \quad\left[H_{k}, L_{k}^{\dagger}\right]=L_{k}^{\dagger}
$$

The operator $N\left(H_{k}\right) \equiv L_{k}^{\dagger} L_{k}$, which generalizes the standard number operator for the harmonic oscillator, is a polynomial in $H_{k}$ of $(2 k+1)$-th order $\left.[79,81,109]\right)$ :

$$
\begin{aligned}
N\left(H_{k}\right) & \equiv L_{k}^{\dagger} L_{k}=B_{k}^{\dagger} a^{\dagger} B_{k} B_{k}^{\dagger} a B_{k}=B_{k}^{\dagger} a^{\dagger} \prod_{i=1}^{k}\left(H_{0}-\epsilon_{i}\right) a B_{k} \\
& =B_{k}^{\dagger}\left(H_{0}-\frac{1}{2}\right) \prod_{i=1}^{k}\left(H_{0}-\epsilon_{i}-1\right) B_{k} \\
& =\left(H_{k}-\frac{1}{2}\right) \prod_{i=1}^{k}\left(H_{k}-\epsilon_{i}-1\right)\left(H_{k}-\epsilon_{i}\right)
\end{aligned}
$$


Thus, the operators $L_{k}, L_{k}^{\dagger}$ and $H_{k}$ generate a polynomial Heisenberg algebra of order $2 k$ :

$$
\left[L_{k}, L_{k}^{\dagger}\right]=N\left(H_{k}+1\right)-N\left(H_{k}\right)
$$

For consistency, when $k=0$ we should get the standard Heisenberg algebra because $L_{0}=a$ and $L_{0}^{\dagger}=a^{\dagger}$. This linear case is indeed recovered from our formulae:

$$
\left[H_{0}, L_{0}\right]=-L_{0} \quad\left[H_{0}, L_{0}^{\dagger}\right]=L_{0}^{\dagger} \quad\left[L_{0}, L_{0}^{\dagger}\right]=I
$$

The corresponding number operator becomes the standard linear expression in terms of the oscillator Hamiltonian:

$$
N\left(H_{0}\right)=H_{0}-\frac{1}{2}=N
$$

On the other hand, when $k=1$ and $\epsilon_{1}$ is arbitrary we recover the quadratic Heisenberg algebra [82] (see also [79,81]):

$$
\left[L_{1}, L_{1}^{\dagger}\right]=\left(H_{1}-\epsilon_{1}\right)\left(3 H_{1}-\epsilon_{1}\right)
$$

The number operator becomes now cubic in $H_{1}$ :

$$
N\left(H_{1}\right)=\left(H_{1}-\frac{1}{2}\right)\left(H_{1}-\epsilon_{1}\right)\left(H_{1}-\epsilon_{1}-1\right)
$$

If $k=2$ we will get a polynomial Heisenberg algebra of fourth order:

$$
\left[L_{2}, L_{2}^{\dagger}\right]=\left(H_{2}-\epsilon_{1}\right)\left(H_{2}-\epsilon_{2}\right)\left[5 H_{2}^{2}-3\left(\epsilon_{1}+\epsilon_{2}\right) H_{2}+\epsilon_{1} \epsilon_{2}+1\right]
$$

where $\mathrm{N}\left(\mathrm{H}_{2}\right)$ is a 5 -th order polynomial of $\mathrm{H}_{2}$ :

$$
N\left(H_{2}\right)=\left(H_{2}-\frac{1}{2}\right)\left(H_{2}-\epsilon_{1}\right)\left(H_{2}-\epsilon_{2}\right)\left(H_{2}-\epsilon_{1}-1\right)\left(H_{2}-\epsilon_{2}-1\right)
$$

For general $k$, it arises a polynomial Heisenberg algebra of order $2 k$ whose properties are characterized by the $(2 k+1)$-th order polynomial $N\left(H_{k}\right)$ of (94).

It should be clear now why the roots of (94) are $\left\{1 / 2, \epsilon_{i}, \epsilon_{i}+1, i=1, \ldots, k\right\}$ : our H-SUSY partner Hamiltonians $H_{k}$ have precisely $k+1$ physical extremal states associated to the $k+1$ roots (eigenvalues) $\left\{1 / 2, \epsilon_{i}, i=1, \ldots, k\right\}$. As the ladder starting from $1 / 2$ is infinite, it does not imply any restriction on the remaining roots of the $(2 k+1)$-th order polynomial $N\left(H_{k}\right)$. However, as the ladders starting from $\epsilon_{i}$ are of length 1 (they end again at the initial energy $\left.\epsilon_{i}\right)$, the other $k$ roots become $\epsilon_{i}+1, i=1, \ldots, k$, as those appearing in (94).

An interesting point to be addressed next, concerning the non-linear nature of the polynomial algebras (92 95), is that they can be partially linearized $[67,85,86]$.

\subsection{Linearization of the non-linear algebra of $H_{k}$}

As pointed out previously, the $k$ eigenstates of $H_{k},\left|\psi_{\epsilon_{i}}^{k}\right\rangle, i=1, \ldots, k$, are isolated between themselves and from the $\left|\psi_{n}^{k}\right\rangle$ because $L_{k}\left|\psi_{\epsilon_{i}}^{k}\right\rangle=0=L_{k}^{\dagger}\left|\psi_{\epsilon_{i}}^{k}\right\rangle$. Hence, it seems natural to look for a linearization on the subspace spanned by $\left\{\left|\psi_{n}^{k}\right\rangle, n=0,1, \ldots\right\}$. The method 
consists in modifying the ladder operators $L_{k}$ and $L_{k}^{\dagger}$ of (92) to get an action similar to the resulting one when the Heisenberg algebra generators are applied to the appropriate energy eigenstates $[67,85,86]$. As for the sub-basis $\left\{\left|\psi_{n}^{k}\right\rangle, n=0,1, \ldots\right\}$ the commutator $\left[L_{k}, L_{k}^{\dagger}\right]$ is already diagonal (see equation (95)), we propose a modification which will convert most of the diagonal elements of $\left[L_{k}, L_{k}^{\dagger}\right]$ to 1 , namely:

$$
L_{L}=B_{k}^{\dagger} f(N) a B_{k} \quad L_{L}^{\dagger}=B_{k}^{\dagger} a^{\dagger} f(N) B_{k}
$$

$N=a^{\dagger} a$ being the standard number operator for the harmonic oscillator, $f(x)$ a real function to be determined and the subscript $L$ denoting linearization. We ask that $\left[L_{L}, L_{L}^{\dagger}\right]=I$ on the subspace $\mathcal{H}_{>1}$ spanned by $\left\{\left|\psi_{n}^{k}\right\rangle, n=1,2, \ldots\right\}$. Notice that we leave open the possibility that $\left[L_{L}, L_{L}^{\dagger}\right]\left|\psi_{0}^{k}\right\rangle=\mathrm{c}\left|\psi_{0}^{k}\right\rangle, \mathrm{c} \neq 1 \in \mathbb{R}$. It is straightforward to show that

$$
\begin{gathered}
L_{L}\left|\psi_{n}^{k}\right\rangle=\sqrt{g(n)}\left|\psi_{n-1}^{k}\right\rangle \quad L_{L}^{\dagger}\left|\psi_{n}^{k}\right\rangle=\sqrt{g(n+1)}\left|\psi_{n+1}^{k}\right\rangle \\
{\left[L_{L}, L_{L}^{\dagger}\right]\left|\psi_{n}^{k}\right\rangle=[g(n+1)-g(n)]\left|\psi_{n}^{k}\right\rangle}
\end{gathered}
$$

where

$$
\begin{aligned}
g(n)= & n[f(n-1)]^{2} \prod_{i=1}^{k}\left(n-\epsilon_{i}-\frac{1}{2}\right)\left(n-\epsilon_{i}+\frac{1}{2}\right) \\
& g(n+1)-g(n)=1 \quad n=1,2, \ldots
\end{aligned}
$$

The general solution of the finite difference equation (104) becomes:

$$
g(n)=n+\mathrm{w}(n)
$$

$\mathrm{w}(n)$ being periodic with period $1, \mathrm{w}(n+1)=\mathrm{w}(n), n=1,2, \ldots$ Hence:

$$
f(n-1)=\sqrt{\frac{n+\mathrm{w}(n)}{n \prod_{i=1}^{k}\left(n-\epsilon_{i}-\frac{1}{2}\right)\left(n-\epsilon_{i}+\frac{1}{2}\right)}}
$$

Since $\mathrm{w}(n)$ takes the same value for all $n=1,2, \ldots$, it is important just $\mathrm{w} \equiv \mathrm{w}(1)$. Moreover, $f(n-1)$ should be real which implies that $\mathrm{w} \geq-1$. Finally, the ladder operators we were looking for read [67]:

$$
\begin{aligned}
& L_{L}=B_{k}^{\dagger} \sqrt{\frac{N+1+\mathrm{w}}{(N+1) \prod_{i=1}^{k}\left(N-\epsilon_{i}+\frac{1}{2}\right)\left(N-\epsilon_{i}+\frac{3}{2}\right)}} a B_{k} \\
& L_{L}^{\dagger}=B_{k}^{\dagger} a^{\dagger} \sqrt{\frac{N+1+\mathrm{w}}{(N+1) \prod_{i=1}^{k}\left(N-\epsilon_{i}+\frac{1}{2}\right)\left(N-\epsilon_{i}+\frac{3}{2}\right)}} B_{k}
\end{aligned}
$$

Although their explicit forms are more involved than the ones for $L_{k}$ and $L_{k}^{\dagger}$ (compare (92) with (107108)), however $L_{L}$ and $L_{L}^{\dagger}$ act simpler on the energy eigenstates $\left|\psi_{n}^{k}\right\rangle, n=0,1, \ldots$ (except by the case with $k=0$ which is separately discussed):

$$
\begin{gathered}
L_{L}\left|\psi_{n}^{k}\right\rangle=\left(1-\delta_{n 0}\right) \sqrt{n+\mathrm{w}}\left|\psi_{n-1}^{k}\right\rangle \quad L_{L}^{\dagger}\left|\psi_{n}^{k}\right\rangle=\sqrt{n+\mathrm{w}+1}\left|\psi_{n+1}^{k}\right\rangle \\
{\left[L_{L}, L_{L}^{\dagger}\right]\left|\psi_{n}^{k}\right\rangle=\left(1+\mathrm{w} \delta_{n 0}\right)\left|\psi_{n}^{k}\right\rangle}
\end{gathered}
$$


This representation is independent of $k$, i.e., of the order of the interwining operator used to go from $H_{0}$ to $H_{k}$. The modified algebra here derived coincides with the 'distorted' Heisenberg algebra originally introduced to linearize partially the second-order Heisenberg algebra characterizing the Abraham-Moses potentials, where $\mathrm{w} \geq-1$ is the distortion parameter [85] (see also [67]). We have shown that this distorted algebra is common to all the H-SUSY partners of the oscillator. Moreover, a 'complete' linearization on $\mathcal{H}_{\geq 0}$ (the subspace spanned by $\left.\left\{\left|\psi_{n}^{k}\right\rangle, n=0,1, \ldots\right\}\right)$ can be achieved by taking $\mathrm{w}=0$ to obtain precisely the Heisenberg algebra representation, namely:

$$
L_{L}\left|\psi_{n}^{k}\right\rangle=\sqrt{n}\left|\psi_{n-1}^{k}\right\rangle \quad L_{L}^{\dagger}\left|\psi_{n}^{k}\right\rangle=\sqrt{n+1}\left|\psi_{n+1}^{k}\right\rangle \quad\left[L_{L}, L_{L}^{\dagger}\right]\left|\psi_{n}^{k}\right\rangle=\left|\psi_{n}^{k}\right\rangle
$$

If $\mathrm{w}=-1$ we get once again the standard Heisenberg algebra on $\mathcal{H}_{\geq 1}$ but the state $\left|\psi_{0}^{k}\right\rangle$ is annihilated by both $L_{L}$ and $L_{L}^{\dagger}$, i.e., it has been isolated by hand of the rest of eigenstates of $H_{k}$. This isolation property is natural for the other $k$ eigenstates $\left|\psi_{\epsilon_{i}}^{k}\right\rangle, i=1, \ldots, k$.

Notice that for $k=0$ the explicit expressions for $L_{L}, L_{L}^{\dagger}$ do not coincide with $a, a^{\dagger}$ :

$$
L_{L}=\sqrt{\frac{N+1+\mathrm{w}}{N+1}} a \quad L_{L}^{\dagger}=a^{\dagger} \sqrt{\frac{N+1+\mathrm{w}}{N+1}}
$$

Now we get a distortion of the Heisenberg algebra representation which maps the operators $a, a^{\dagger}$ into $L_{L}, L_{L}^{\dagger}$, by changing the matrix elements of $a$ and $a^{\dagger}$ without affecting the diagonal elements of $\left[a, a^{\dagger}\right]$ except the one associated to $\left|\psi_{0}^{0}\right\rangle$, which becomes $\mathrm{w}+1$. For $\mathrm{w}=0$ we recover the original Heisenberg algebra because now $L_{L}=a, L_{L}^{\dagger}=a^{\dagger}$. Moreover, when $\mathrm{w}=-1$ we get a reducible representation composed of the Heisenberg algebra on $\mathcal{H}_{\geq 1}$ and the null representation on the subspace generated by $\left|\psi_{0}^{0}\right\rangle$ due to $L_{L}\left|\psi_{0}^{0}\right\rangle=L_{L}^{\dagger}\left|\psi_{0}^{0}\right\rangle=0$. 


\section{Coherent states for the SUSY partners of the oscil- lator}

The beautiful properties of the harmonic oscillator coherent states (CS) motivated the interest in looking for them in other physical situations [84-94,110-120]. There are various definitions, each one of them leading in general to different sets of CS. Concerning the intertwining technique, CS which are eigenstates of a certain annihilation operator for the shape invariant potentials were constructed by Fukui and Aizawa [87]. The CS as eigenstates of the annihilation operator $L_{k}$ of (92) with $k=1$ and $\epsilon=-1 / 2$, i.e. for the Abraham-Moses family of isospectral oscillator potentials were derived in 1994 [84], and the linearization process in the same case as well as the corresponding CS analysis was elaborated in [85,86]. Since then, a lot of works have arisen looking for interrelations between CS and quantum groups, pseudodifferential operators, non-linear algebras, etcetera [88-94,118, 119]. In particular, the CS construction for the H-SUSY partners of the oscillator with arbitrary $k$ has been successfully addressed [67]. For didactic purposes, let us discuss first the main properties of the standard CS [110-114].

\subsection{Standard coherent states}

There are three equivalent definitions of the harmonic oscillator coherent states which can be used to define them in other physical situations.

1. The coherent states $|z\rangle$ are eigenstates of the annihilation operator

$$
a|z\rangle=z|z\rangle \quad z \in \mathbb{C}
$$

2. The coherent states $|z\rangle$ are obtained by applying the displacement operator $D(z)$ onto the oscillator ground state $|0\rangle$ (in this subsection we use the standard notation (Fock) for the eigenstates of the number operator, i.e., $\left.|n\rangle \equiv\left|\psi_{n}^{0}\right\rangle\right)$ :

$$
|z\rangle=D(z)|0\rangle \quad D(z)=e^{z a^{\dagger}-\bar{z} a}
$$

3. The coherent states $|z\rangle$ are quantum states with a minimum-uncertainty relationship

$$
(\Delta x)(\Delta p)=\frac{1}{2}
$$

where for a system in the state $|z\rangle$ and an arbitrary operator $\mathcal{O}$ the corresponding uncertainty expresses:

$$
\Delta \mathcal{O}=\sqrt{\left\langle z\left|(\mathcal{O}-\langle z|\mathcal{O}| z\rangle)^{2}\right| z\right\rangle}=\sqrt{\left\langle z\left|\mathcal{O}^{2}\right| z\right\rangle-\langle z|\mathcal{O}| z\rangle^{2}}
$$

Since for general systems we are specially interested in the first definition, we derive here the standard CS through (113). First we expand $|z\rangle$ in the Fock basis

$$
|z\rangle=\sum_{n=0}^{\infty} c_{n}|n\rangle
$$


Then we use (113) to obtain:

$$
a|z\rangle=\sum_{n=1}^{\infty} c_{n} \sqrt{n}|n-1\rangle=\sum_{n=0}^{\infty} z c_{n}|n\rangle=\sum_{n=1}^{\infty} z c_{n-1}|n-1\rangle
$$

Hence, we get a recursion formula for $c_{n}$ :

$$
c_{n}=\frac{z c_{n-1}}{\sqrt{n}}
$$

By iterating this equation we arrive at:

$$
c_{n}=\frac{z^{n} c_{0}}{\sqrt{n !}}
$$

Finally, by asking that $c_{0}$ is a positive constant such that $\langle z \mid z\rangle=1$ we get:

$$
|z\rangle=e^{-\frac{r^{2}}{2}} \sum_{n=0}^{\infty} \frac{z^{n}}{\sqrt{n !}}|n\rangle
$$

where $r=|z|$. Let us remark that the standard coherent states can be used as an alternative basis (non-orthogonal) in the Hilbert space of states because they form a complete set:

$$
\frac{1}{\pi} \int|z\rangle\langle z| d^{2} z=I
$$

This can be straightforwardly verified since in the polar representation $z=r e^{i \theta}$ we have:

$$
\begin{aligned}
\frac{1}{\pi} \int|z\rangle\langle z| d^{2} z & =\frac{1}{\pi} \sum_{n, m=0}^{\infty} \frac{|n\rangle\langle m|}{\sqrt{n ! m !}} \int_{0}^{\infty} e^{-r^{2}} r^{n+m+1} d r \int_{0}^{2 \pi} e^{i \theta(n-m)} d \theta \\
& =\sum_{n=0}^{\infty} \frac{|n\rangle\langle n|}{n !} 2 \int_{0}^{\infty} e^{-r^{2}} r^{2 n+1} d r=\sum_{n=0}^{\infty}|n\rangle\langle n|=I
\end{aligned}
$$

Thus, any state can be expanded in the basis of coherent states. In particular, any CS $\left|z^{\prime}\right\rangle$ admits a non-trivial decomposition:

$$
\left|z^{\prime}\right\rangle=\frac{1}{\pi} \int|z\rangle\left\langle z \mid z^{\prime}\right\rangle d^{2} z
$$

where the reproducing Kernel $\left\langle z \mid z^{\prime}\right\rangle$ is given by

$$
\left\langle z \mid z^{\prime}\right\rangle=e^{-\frac{r^{2}}{2}+\bar{z} z^{\prime}-\frac{r^{\prime 2}}{2}}
$$

Let us notice that coherent states evolve into coherent states, namely

$$
e^{-i t H}|z\rangle=e^{-\frac{r^{2}}{2}} \sum_{n=0}^{\infty} \frac{z^{n}}{\sqrt{n !}} e^{-i\left(n+\frac{1}{2}\right) t}|n\rangle=e^{-i \frac{t}{2}}|z(t)\rangle \quad z(t)=z e^{-i t}
$$




\subsection{Coherent states for $H_{k}$}

Let us construct the CS for the SUSY partner Hamiltonians $H_{k}$ of the oscillator as eigenstates of the annihilation operators $L_{k}$ and $L_{L}$. First, let us find them as eigenstates of $L_{k}$ (the non-linear case):

$$
L_{k}|z\rangle=z|z\rangle \quad z \in \mathbb{C}
$$

We express $|z\rangle$ as a linear combination of the subset of eigenstates $\left|\psi_{n}^{k}\right\rangle$ of $H_{k}$ associated to the part of the spectrum isospectral to the oscillator:

$$
|z\rangle=\sum_{n=0}^{\infty} c_{n}\left|\psi_{n}^{k}\right\rangle
$$

After inserting (126) in (125) and using the fact that

$$
L_{k}\left|\psi_{n}^{k}\right\rangle=\sqrt{n \prod_{i=1}^{k}\left(n-\epsilon_{i}-\frac{1}{2}\right)\left(n-\epsilon_{i}+\frac{1}{2}\right)}\left|\psi_{n-1}^{k}\right\rangle
$$

we get a recurrence relationship for the coefficients $c_{n}$

$$
c_{n}=\frac{z c_{n-1}}{\sqrt{n \prod_{i=1}^{k}\left(n-\epsilon_{i}-\frac{1}{2}\right)\left(n-\epsilon_{i}+\frac{1}{2}\right)}}=\frac{\sqrt{\prod_{i=1}^{k} \Gamma\left(-\epsilon_{i}+\frac{1}{2}\right) \Gamma\left(-\epsilon_{i}+\frac{3}{2}\right)} z^{n} c_{0}}{\sqrt{n ! \prod_{i=1}^{k} \Gamma\left(n-\epsilon_{i}+\frac{1}{2}\right) \Gamma\left(n-\epsilon_{i}+\frac{3}{2}\right)}}
$$

We fix $c_{0}$ by the condition $\langle z \mid z\rangle=1$ and asking that $c_{0}>0$. Hence, our non-linear CS become:

$$
|z\rangle=\sum_{n=0}^{\infty} \frac{\sqrt{\prod_{i=1}^{k} \Gamma\left(-\epsilon_{i}+\frac{1}{2}\right) \Gamma\left(-\epsilon_{i}+\frac{3}{2}\right)} z^{n}\left|\psi_{n}^{k}\right\rangle}{\sqrt{n !_{0} F_{2 k}\left(-\epsilon_{1}+\frac{1}{2}, \ldots,-\epsilon_{k}+\frac{1}{2},-\epsilon_{1}+\frac{3}{2}, \ldots,-\epsilon_{k}+\frac{3}{2} ; r^{2}\right) \prod_{i=1}^{k} \Gamma\left(n-\epsilon_{i}+\frac{1}{2}\right) \Gamma\left(n-\epsilon_{i}+\frac{3}{2}\right)}}
$$

being $\Gamma(x)$ the gamma function, $r=|z|$, and ${ }_{p} F_{q}$ a generalized hypergeometric function:

$$
{ }_{p} F_{q}\left(a_{1}, \ldots, a_{p}, b_{1}, \ldots, b_{q} ; x\right)=\frac{\Gamma\left(b_{1}\right) \ldots \Gamma\left(b_{q}\right)}{\Gamma\left(a_{1}\right) \ldots \Gamma\left(a_{p}\right)} \sum_{n=0}^{\infty} \frac{\Gamma\left(a_{1}+n\right) \ldots \Gamma\left(a_{p}+n\right)}{\Gamma\left(b_{1}+n\right) \ldots \Gamma\left(b_{q}+n\right)} \frac{x^{n}}{n !}
$$

Notice that $z=0$ is a $(k+1)$-th degenerate eigenvalue of $L_{k}$ because we get of (129) that $|z=0\rangle=\left|\psi_{0}^{k}\right\rangle$ and $L_{k}\left|\psi_{\epsilon_{i}}^{k}\right\rangle=0, i=1, \ldots, k$. Thus, the resolution of the identity should be looked for as:

$$
I=\sum_{i=1}^{k}\left|\psi_{\epsilon_{i}}^{k}\right\rangle\left\langle\psi_{\epsilon_{i}}^{k}\left|+\int\right| z\right\rangle\langle z| d \mu(z)
$$

where the measure $d \mu(z)$ is to be determined. Suppose now that

$$
d \mu(z)={ }_{0} F_{2 k}\left(-\epsilon_{1}+\frac{1}{2}, \ldots,-\epsilon_{k}+\frac{1}{2},-\epsilon_{1}+\frac{3}{2}, \ldots,-\epsilon_{k}+\frac{3}{2} ; r^{2}\right) h\left(r^{2}\right) r d r d \theta
$$

Inserting this equation in (131) and using the fact that $\left\{\left|\psi_{\epsilon_{i}}^{k}\right\rangle,\left|\psi_{n}^{k}\right\rangle, i=1, \ldots, k, n=\right.$ $0,1, \ldots\}$ is complete, we arrive at the following requirement for $h(x)$ :

$$
\int_{0}^{\infty} x^{n} h(x) d x=\frac{\Gamma(n+1) \prod_{i=1}^{k} \Gamma\left(n-\epsilon_{i}+\frac{1}{2}\right) \Gamma\left(n-\epsilon_{i}+\frac{3}{2}\right)}{\pi \prod_{i=1}^{k} \Gamma\left(-\epsilon_{i}+\frac{1}{2}\right) \Gamma\left(-\epsilon_{i}+\frac{3}{2}\right)}
$$


Hence, $h(x)$ is the inverse Mellin transform of the right hand side of (133) [121]. It turns out that $h(x)$ is proportional to a Meijer $G$-function $[67,121,122]$ :

$$
h(x)=\frac{G_{02 k+1}^{2 k+1} \operatorname{lo}_{0}\left(x \mid 0,-\epsilon_{1}-\frac{1}{2}, \ldots,-\epsilon_{k}-\frac{1}{2},-\epsilon_{1}+\frac{1}{2}, \ldots,-\epsilon_{k}+\frac{1}{2}\right)}{\pi \prod_{i=1}^{k} \Gamma\left(-\epsilon_{i}+\frac{1}{2}\right) \Gamma\left(-\epsilon_{i}+\frac{3}{2}\right)}
$$

Let us notice that for $k=1$ and $\epsilon_{1}=-1 / 2$ an explicit expression for (134) has been derived in [84].

The other properties of the standard coherent states have their corresponding analogue here. For instance, any CS of the form (129) can be expressed in terms of the others:

$$
\left|z^{\prime}\right\rangle=\int|z\rangle\left\langle z \mid z^{\prime}\right\rangle d \mu(z)
$$

where the reproducing Kernel $\left\langle z \mid z^{\prime}\right\rangle$ can be straightforwardly evaluated:

$$
\left\langle z \mid z^{\prime}\right\rangle=\frac{{ }_{0} F_{2 k}\left(-\epsilon_{1}+\frac{1}{2}, \ldots,-\epsilon_{k}+\frac{1}{2},-\epsilon_{1}+\frac{3}{2}, \ldots,-\epsilon_{k}+\frac{3}{2} ; \bar{z} z^{\prime}\right)}{\sqrt{{ }_{0} F_{2 k}\left(-\epsilon_{1}+\frac{1}{2}, \ldots,-\epsilon_{k}+\frac{1}{2},-\epsilon_{1}+\frac{3}{2}, \ldots,-\epsilon_{k}+\frac{3}{2} ; r^{2}\right)_{0} F_{2 k}\left(-\epsilon_{1}+\frac{1}{2}, \ldots,-\epsilon_{k}+\frac{1}{2},-\epsilon_{1}+\frac{3}{2}, \ldots,-\epsilon_{k}+\frac{3}{2} ; r^{\prime 2}\right)}}
$$

This means that any two CS $|z\rangle$ and $\left|z^{\prime}\right\rangle$ of form (129) are non-orthogonal. From the resolution of the identity (131) it is clear that any state vector can be expanded in terms of our CS if we include the atypical orthogonal CS $\left|\psi_{\epsilon_{i}}^{k}\right\rangle, i=1, \ldots, k$ naturally inherent to this treatment. Notice also that our CS evolve in time as coherent states:

$$
U(t)|z\rangle=e^{-\frac{i t}{2}}|z(t)\rangle \quad z(t)=z e^{-i t}
$$

Let us derive now the coherent states for the linearized annihilation operator $L_{L}$ of (107) as:

$$
L_{L}|z, \mathrm{w}\rangle=z|z, \mathrm{w}\rangle
$$

where we have made explicit the CS dependence on the distortion parameter $\mathrm{w}$. The expansion of $|z, \mathrm{w}\rangle$ in the sub-basis $\left|\psi_{n}^{k}\right\rangle$ is equal to the one arising in the right hand side of (126), and the use of (137) leads to the following recurrence relationship for the $c_{n}$ 's:

$$
c_{n}=\frac{z c_{n-1}}{\sqrt{n+\mathrm{w}}}=\frac{\sqrt{\Gamma(\mathrm{w}+1)} z^{n}}{\Gamma(n+\mathrm{w}+1)} c_{0}
$$

The coefficient $c_{0}$ is determined by asking that $\langle z, \mathrm{w} \mid z, \mathrm{w}\rangle=1$ and $c_{0}>0$. Finally

$$
|z, \mathrm{w}\rangle=\sqrt{\frac{\Gamma(\mathrm{w}+1)}{{ }_{1} F_{1}\left(1, \mathrm{w}+1 ; r^{2}\right)}} \sum_{n=0}^{\infty} \frac{z^{n}}{\sqrt{\Gamma(n+\mathrm{w}+1)}}\left|\psi_{n}^{k}\right\rangle
$$

The resolution of the identity becomes similar to (131):

$$
I=\sum_{i=1}^{k}\left|\psi_{\epsilon_{i}}^{k}\right\rangle\left\langle\psi_{\epsilon_{i}}^{k}\left|+\int\right| z, \mathrm{w}\right\rangle\langle z, \mathrm{w}| d \mu_{L}(z)
$$


where the measure $d \mu_{L}(z)$ is given by:

$$
d \mu_{L}(z)=\sigma(r, \mathrm{w}) r d r d \theta \quad \sigma(r, \mathrm{w})=\frac{{ }_{1} F_{1}\left(1, \mathrm{w}+1 ; r^{2}\right)}{\pi \Gamma(\mathrm{w}+1)} e^{-r^{2}} r^{2 \mathrm{w}}
$$

The reproducing Kernel is now

$$
\left\langle z, \mathrm{w} \mid z^{\prime}, \mathrm{w}\right\rangle=\frac{{ }_{1} F_{1}\left(1, \mathrm{w}+1 ; \bar{z} z^{\prime}\right)}{\sqrt{{ }_{1} F_{1}\left(1, \mathrm{w}+1 ; r^{2}\right){ }_{1} F_{1}\left(1, \mathrm{w}+1 ; r^{\prime 2}\right)}}
$$

For $\mathrm{w}=0$ (the linearized case in $\mathcal{H}_{\geq 0}$ ) the same formulae as for the standard coherent states are recovered by noticing that ${ }_{1} F_{1}\left(1,1 ; r^{2}\right)=e^{r^{2}}$. Moreover, by taking carefully the limit $\mathrm{w} \rightarrow-1$ (the linearized case now in $\mathcal{H}_{\geq 1}$ ) it can be shown that the standard expression for the CS is also recovered, but the eigenstate $\left|\psi_{0}^{k}\right\rangle$ associated to the eigenvalue $E_{0}=1 / 2$ will be isolated of the other ones, i.e., the series (138) will start from $\left|\psi_{1}^{k}\right\rangle[67,85,86]$.

A comparison of the annihilation operators $L_{k}$ and $L_{L}$ and of both sets of coherent states derived in this section shows the following: the explicit expression for the non-linear operator $L_{k}$ is simpler than the one for $L_{L}$. As can be seen from equations (129) and (138), however, the CS associated to $L_{L}$ are less involved than the ones associated to $L_{k}$, which is due to the simplest algebra representation generated by $L_{L}$ and $L_{L}^{\dagger}$.

Let us analyze, for both kinds of CS, the uncertainty products $(\Delta x)(\Delta p)$ in cases for which we get closed analytic expressions. This can be directly achieved if we restrict ourselves to H-SUSY partner potentials in the harmonic oscillator limit. In particular, for $k=1$ and $\epsilon_{1}=-1 / 2$ the uncertainty product for the non-linear CS (129) was calculated for the Abraham-Moses potentials in the oscillator limit [84], i.e., by taking $\nu=0$ in (89). We shall present this case as well as the non-linear one with $k=2, \epsilon_{1}=-1 / 2, \epsilon_{2}=-3 / 2$, and taking $\nu_{1}=0, \nu_{2} \rightarrow \infty$ in order to recover the oscillator limit. For the linearized coherent states we shall study the case with $k=1, \epsilon_{1}=-1 / 2, \nu_{1}=0, \mathrm{w}=1$ (which was analyzed in [85, 86]) as well as the one with $k=2, \epsilon_{1}=-1 / 2, \epsilon_{2}=-3 / 2, \nu_{1}=0, \nu_{2} \rightarrow \infty, \mathrm{w}=2$. Notice that the corresponding expressions for the CS can be obtained from equations (129) and (138) by realizing that in the oscillator limit $\left|\psi_{n}^{2}\right\rangle \rightarrow\left|\psi_{n+2}^{0}\right\rangle$ and $\left|\psi_{n}^{1}\right\rangle \rightarrow\left|\psi_{n+1}^{0}\right\rangle$. Henceforth, the sets of coherent states we are dealing with read:

$$
\begin{array}{cc}
|z\rangle=\frac{1}{\sqrt{{ }_{0} F_{2}\left(1,2 ; r^{2}\right)}} \sum_{n=0}^{\infty} \frac{z^{n}}{n ! \sqrt{(n+1) !}}\left|\psi_{n+1}^{0}\right\rangle & k=1 \\
|z\rangle=\sqrt{\frac{2}{{ }_{0} F_{4}\left(1,2,2,3 ; r^{2}\right)}} \sum_{n=0}^{\infty} \frac{z^{n}}{n !(n+1) ! \sqrt{(n+2) !}}\left|\psi_{n+2}^{0}\right\rangle & k=2 \\
|z, \mathrm{~W}=1\rangle=\frac{1}{\sqrt{1 F_{1}\left(1,2 ; r^{2}\right)}} \sum_{n=0}^{\infty} \frac{z^{n}}{\sqrt{(n+1) !}}\left|\psi_{n+1}^{0}\right\rangle & k=1 \\
|z, \mathrm{~W}=2\rangle=\sqrt{\frac{2}{1 F_{1}\left(1,3 ; r^{2}\right)}} \sum_{n=0}^{\infty} \frac{z^{n}}{\sqrt{(n+2) !}}\left|\psi_{n+2}^{0}\right\rangle & k=2
\end{array}
$$

A direct calculation using (142) and $x=\left(a+a^{\dagger}\right) / \sqrt{2}, p=i\left(a^{\dagger}-a\right) / \sqrt{2}$ leads to the 


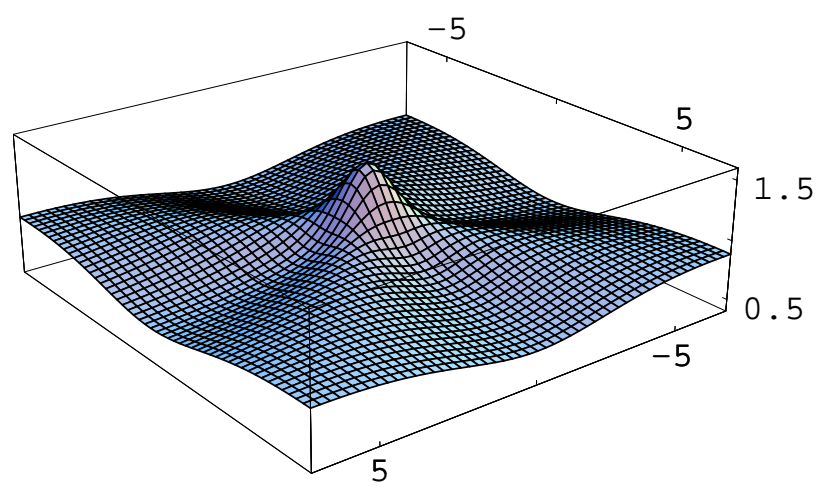

Figure 9: The uncertainty product $(\Delta x)(\Delta p)$ (vertical axis) as function of $z$ for the non-linear coherent states (142) associated to the oscillator

uncertainties $\Delta x$ and $\Delta p$ in the non-linear case for $k=1$ :

$$
\begin{aligned}
& \Delta x=\sqrt{\frac{3}{2}-[\operatorname{Re}(z)]^{2} \rho_{1}(r)} \\
& \Delta p=\sqrt{\frac{3}{2}-[\operatorname{Im}(z)]^{2} \rho_{1}(r)} \\
& \rho_{1}(r)=2\left[\frac{{ }_{0} F_{2}\left(2,2 ; r^{2}\right)}{{ }_{0} F_{2}\left(1,2 ; r^{2}\right)}\right]^{2}-\frac{{ }_{0} F_{2}\left(2,3 ; r^{2}\right)}{{ }_{0} F_{2}\left(1,2 ; r^{2}\right)}
\end{aligned}
$$

where $\operatorname{Re}(z)$ and $\operatorname{Im}(z)$ represent the real and imaginary parts of $z$ respectively. A plot of the corresponding uncertainty product is given in figure 9 .

On the other hand, for $k=2$ and employing the non-linear CS in (143) it is found that:

$$
\begin{aligned}
& \Delta x=\sqrt{\frac{5}{2}-[\operatorname{Re}(z)]^{2} \rho_{2}(r)} \\
& \Delta p=\sqrt{\frac{5}{2}-[\operatorname{Im}(z)]^{2} \rho_{2}(r)} \\
& \rho_{2}(r)=\frac{1}{2}\left[\frac{{ }_{0} F_{4}\left(2,2,3,3 ; r^{2}\right)}{{ }_{0} F_{4}\left(1,2,2,3 ; r^{2}\right)}\right]^{2}-\frac{1}{6}\left[\frac{{ }_{0} F_{4}\left(2,3,3,4 ; r^{2}\right)}{{ }_{0} F_{4}\left(1,2,2,3 ; r^{2}\right)}\right]
\end{aligned}
$$

A plot of $(\Delta x)(\Delta p)$ is given in figure 10 .

On the other hand, in the linearized case with $k=1$ and making use of the CS in (144) we get:

$$
(\Delta x)^{2}=(\Delta p)^{2}=(\Delta x)(\Delta p)=\frac{1}{2}+\frac{1}{{ }_{1} F_{1}\left(1,2 ; r^{2}\right)}
$$

A plot of the uncertainty product is given in figure 11 .

Finally, in the linearized case with $k=2$ and employing (145) we find:

$$
(\Delta x)^{2}=(\Delta p)^{2}=(\Delta x)(\Delta p)=\frac{1}{2}+\frac{2}{{ }_{1} F_{1}\left(1,3 ; r^{2}\right)}
$$




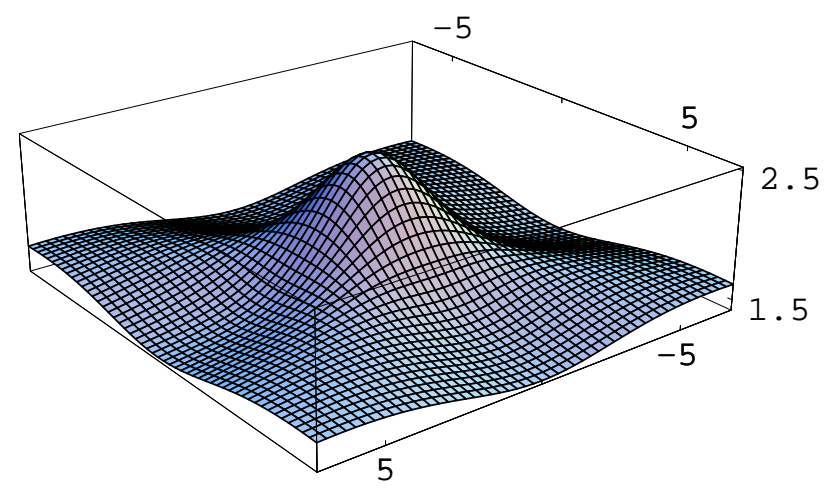

Figure 10: The uncertainty product $(\Delta x)(\Delta p)$ (vertical axis) as function of $z$ for the nonlinear coherent states (143) associated to the oscillator

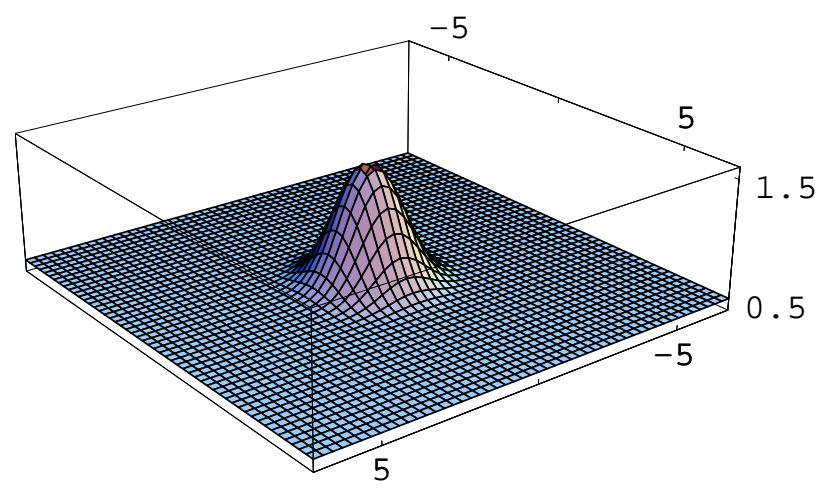

Figure 11: The uncertainty product $(\Delta x)(\Delta p)$ (vertical axis) as function of $z$ for the linearized coherent states (144) of the oscillator

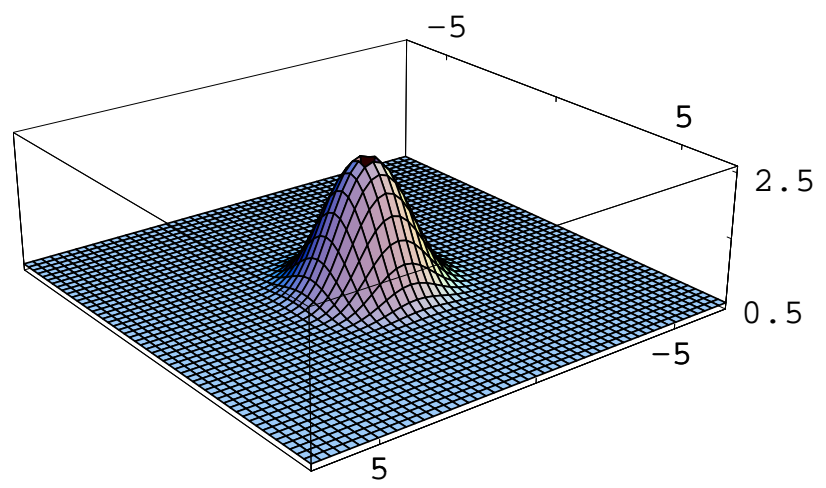

Figure 12: The uncertainty product $(\Delta x)(\Delta p)$ (vertical axis) as function of $z$ for the linearized coherent states (145) of the oscillator 
A plot of $(\Delta x)(\Delta p)$ is given in figure 12 .

As we can see, $(\Delta x)(\Delta p)$ have more structure in the non-linear than in the linearized cases. Indeed, in the linear cases $(\Delta x)(\Delta p)$ have radial symmetry, they differ of the standard result $(\Delta x)(\Delta p)=1 / 2$ just in a neighborhood about the origin, and they quickly approach the standard value when $|z| \rightarrow \infty$ (see figure 11 and 12). This does not happens for the non-linear CS for which the asymptotic values of $(\Delta x)(\Delta p)$ are in general different from $1 / 2$ and depend on the direction in which we move out of $z=0$ (see figures 9 and 10).

We conclude that the annihilation and creation operators for the oscillator H-SUSY partner Hamiltonians which are more similar to the standard oscillator ones become $L_{L}$ and $L_{L}^{\dagger}$. They mimic quite well the annihilation and creation operators of the oscillator and lead to the standard CS expression in the cases when the distortion parameter takes the two values $\mathrm{w}=0$ and $\mathrm{w}=-1$. Moreover, $L_{L}$ and $L_{L}^{\dagger}$ become exactly equal to $a$ and $a^{\dagger}$ when $k=\mathrm{w}=0$, and the corresponding CS are precisely the standard ones for the oscillator. 


\section{Conclusions and outlook}

We have discussed the possibilities for designing quantum spectra offered by the supersymmetric quantum mechanics. We have seen that the standard iterative method, in which the first-order SUSY transformations are used to construct higher-order ones, could induce the wrong conclusion that the new Hamiltonians will have always the new levels below the ground state energy of the initial one. Here, it has been shown that the direct procedure frees us of that belief, allowing to create under certain restrictions some new levels above the initial ground state energy. It is important to underline also the complex SUSY transformations which can be employed to generate non-hermitian Hamiltonians with either purely real spectra or with some finite number of complex levels [102-104]. We think that this interesting line of research is worth to be continued.

When the SUSY techniques are applied to the harmonic oscillator, some interrelations immediately arise with several subjects of mathematical physics as non-linear deformations of Lie algebras and coherent states [67]. It can be established also connections between SUSY techniques and non-linear ordinary differential equations as Riccati [97], Painlevé [75] and $\mathrm{KdV}$ [56]. In particular, when looking for the general systems ruled by secondorder polynomial Heisenberg algebras, it turns out that the corresponding potentials become determined by a certain function which obeys the Painlevé IV equation $[107,109]$. It has been recently shown that subsets of H-SUSY partner Hamiltonians of the oscillator for which the $k$ levels $\epsilon_{i}, i=k, \ldots, 1$ are forced to be connected by appropriate annihilation and creation operators to form a finite ladder of equally spaced levels, supply us with explicit solutions of the Painlevé IV equations [123]. Notice that a similar treatment relates subsets of SUSY partner potentials of the radial oscillator and solutions of the Painlevé V equation [75].

We conclude by mentioning that links of this kind between SUSY techniques and nonlinear aspects of mathematical-physics, as the ones previously pointed out, represent the future of a field which has proved very fruitful along the years (see e.g. [78]).

Acknowledgements. The authors acknowledge the support of CONACYT (México), project No. 40888-F. 


\section{References}

[1] E. Schrödinger, Proc. Roy. Irish Acad. A 46 (1940) 183

[2] E. Schrödinger, Proc. Roy. Irish Acad. A 47 (1941) 53

[3] L. Infeld, T.E. Hull, Rev. Mod. Phys. 23 (1951) 21

[4] E. Witten, Nucl. Phys. B 188 (1981) 513

[5] L.F. Urrutia, E. Hernández, Phys. Rev. Lett. 51 (1983) 755

[6] B. Mielnik, J. Math. Phys. 25 (1984) 3387

[7] D.J. Fernández, Lett. Math. Phys. 8 (1984) 337

[8] M.M. Nieto, Phys. Lett. 145B (1984) 208

[9] A.A. Andrianov, N.B. Borisov, M.V. Ioffe, Phys. Lett. 105A (1984) 19

[10] C.V. Sukumar, J. Phys. A 18 (1985) L57

[11] C.V. Sukumar, J. Phys. A 18 (1985) 2917

[12] C.V. Sukumar, J. Phys. A 18 (1985) 2937

[13] C.V. Sukumar, J. Phys. A 19 (1986) 2297

[14] C.V. Sukumar, J. Phys. A 20 (1987) 2461

[15] A. Frank, K.B. Wolf, J. Math. Phys. 26 (1985) 973

[16] Z. Dongpei, J. Phys. A 20 (1987) 4331

[17] J. Beckers, D. Dehin, V. Hussin, J. Phys. A 20 (1987) 1137

[18] J. Beckers, D. Dehin, V. Hussin, J. Phys. A 21 (1988) 651

[19] N.A. Alves, E. Drigo-Filho, J. Phys. A 21 (1988) 3215

[20] E. Drigo-Filho, J. Phys. A 21, L1025 (1988)

[21] L.J. Boya, Eur. J. Phys. 9 (1988) 139

[22] E. Drigo-Filho, R.M. Riccota, Mod. Phys. Lett. A 4 (1989) 2283

[23] G. Levai, J. Phys. A 22 (1989) 689

[24] A. Stahlhofen, K. Bleuler, Nuov. Cim. 104B (1989) 447

[25] A. Arai, Lett. Math. Phys. 19 (1990) 217

[26] M. Moreno, R. Martínez, A. Zentella, Mod. Phys. Lett. A 5 (1990) 949 
[27] O. Castaños, A. Frank, R. López, L.F. Urrutia, Phys. Rev. D 43 (1991) 544

[28] O.L. de Lange, Am. J. Phys. 59 (1991) 151

[29] O.L. de Lange, R.E. Raab, Operator methods in quantum mechanics, Clarendon Press, Oxford (1991)

[30] R.D. Amado, R. Bijker, F. Cannata, J.P. Dedonder, Phys. Rev. Lett. 67 (1991) 2777

[31] J. Casahorrán, S. Nam, Int. J. Mod. Phys. A 6 (1991) 2729

[32] E. Drigo-Filho, Mod. Phys. Lett. A 8 (1993) 63

[33] E. Drigo-Filho, Mod. Phys. Lett. A 10 (1995) 1613

[34] M. Moshinsky, C. Quesne, Y.F. Smirnov, J. Phys. A 28 (1995) 6447

[35] A. Cabo, J.L. Lucio, M. Napsuciale, Ann. Phys. 244 (1995) 1

[36] F. Cooper, A. Khare, U. Sukhatme, Phys. Rep. 251 (1995) 267

[37] H.C. Rosu, M.A. Reyes, Phys. Rev. E 51 (1995) 5112

[38] D.J. Fernández, J. Negro, M.A. del Olmo, Ann. Phys. 252 (1996) 386

[39] A. Pérez-Lorenzana, Rev. Mex. Fis. 42 (1996) 1060

[40] J. Casahorrán, Rev. Math. Phys. 8 (1996) 655

[41] H.C. Rosu, J. Socorro, O. Obregón, J. Phys. A 29 (1996) 1825

[42] V.M. Chabanov, B.N. Zakhariev, Inv. Probl. 13 (1997) R47

[43] G. Junker, P. Roy, Phys. Lett. A 232 (1997) 155

[44] G. Junker, P. Roy, Ann. Phys. 270 (1998) 155

[45] J.F. Cariñena, G. Marmo, A.M. Perelomov, M.F. Rañada, Int. J. Mod. Phys. A 13 (1998) 4913

[46] I.F. Márquez, J. Negro, L.M. Nieto, J. Phys. A 31 (1998) 4115

[47] J. Morales, J.J. Peña, G. Ovando, V. Gaftoi, Int. J. Quant. Chem. 71 (1999) 465

[48] J.F. Cariñena, A. Ramos, Int. J. Mod. Phys. A 14 (1999) 1935

[49] J.F. Cariñena, A. Ramos, Rev. Math. Phys. 12 (2000) 1279

[50] J. Negro, L.M. Nieto, O. Rosas-Ortiz, J. Math. Phys. 41 (2000) 7964

[51] B.K. Bagchi, Supersymmetry in quantum and classical mechanics, Chapman \& Hall, New York (2001) 
[52] R. de Lima Rodrigues, Preprint hep-th/0205017 (2002)

[53] L.M. Nieto, A.A. Pecheritsin, B.F. Samsonov, Ann. Phys. 305 (2003) 151

[54] R.D. Mota, V.D. Granados, A. Queijeiro, J. García, L. Guzmán, J. Phys. A 36 (2003) 4849

[55] J. Socorro, M.A. Reyes, F.A. Gelbert, Phys. Lett. A 313 (2003) 338

[56] B. Mielnik, O. Rosas-Ortiz, J. Phys. A. 37 (2004) 10007

[57] P.A. Deift, Duke Math. J. 45 (1978) 267

[58] R.W. Carrol, Transmutation and Operator Differential Equations, North-Holland Mathematics Studies 37, The Netherlands (1979)

[59] A.A. Andrianov, M.V. Ioffe, V. Spiridonov, Phys. Lett. A 174 (1993) 273

[60] A.A. Andrianov, M.V. Ioffe, F. Cannata, J.P. Dedonder, Int. J. Mod. Phys. A 10 (1995) 2683

[61] V.G. Bagrov, B.F. Samsonov, Phys. Part. Nucl. 28 (1997) 374

[62] D.J. Fernández, Int. J. Mod. Phys. A 12 (1997) 171

[63] D.J. Fernández, M.L. Glasser, L.M. Nieto, Phys. Lett. A 240 (1998) 15

[64] D.J. Fernández, V. Hussin, B. Mielnik, Phys. Lett. A 244 (1998) 309

[65] J. Oscar Rosas-Ortiz, J. Phys. A 31 (1998) L507

[66] J. Oscar Rosas-Ortiz, J. Phys. A 31 (1998) 10163

[67] D.J. Fernández, V. Hussin, J. Phys. A 32 (1999) 3603

[68] B. Bagchi, A. Ganguly, D. Bhaumik, A. Mitra, Mod. Phys. Lett. A 14 (1999) 27

[69] B. Mielnik, L.M. Nieto, O. Rosas-Ortiz, Phys. Lett. A 269 (2000) 70

[70] M. Plyushchay, Int. J. Mod. Phys. A 15 (2000) 3679

[71] D.J. Fernández, H.C. Rosu, Phys. Scripta 64 (2001) 177

[72] H. Aoyama, N. Nakayama, M. Sato, T. Tanaka, Phys. Lett. B 521 (2001) 400

[73] D.Y. Song, J.R. Klauder, J. Phys. A 36 (2003) 8673

[74] M. Plyushchay, J. Phys. A 37 (2004) 10375

[75] J.M. Carballo, D.J. Fernández, J. Negro, L.M. Nieto, J. Phys. A 37 (2004) 10349

[76] A.A. Andrianov, F. Cannata, J. Phys. A 37 (2004) 10297 
[77] D.J. Fernández, A. Ramos in Topics in mathematical physics, general relativity and cosmology on the 75th birthday of Jerzy Plebañski (to be published)

[78] I. Areféva, D.J Fernández, V. Hussin, J. Negro, L.M. Nieto, B.F. Samsonov Eds., special issue Progress in supersymmetric quantum mechanics, J. Phys. A 37, Number $43(2004)$

[79] S.Y. Dubov, V.M. Eleonsky, N.E. Kulagin, Sov. Phys. JETP 75 (1992) 446

[80] V.E. Adler, Physica D 73 (1994) 335

[81] V.M. Eleonsky, V.G. Korolev, J. Phys. A 28 (1995) 4973

[82] N. Aizawa, H.T. Sato, Prog. Theor. Phys. 98 (1997) 707

[83] M. Arik, N.M. Atakishiyev, K.B. Wolf, J. Phys. A 32 (1999) L371

[84] D.J. Fernández, V. Hussin, L.M. Nieto, J. Phys. A 27 (1994) 3547

[85] D.J. Fernández, L.M. Nieto, O. Rosas-Ortiz, J. Phys. A 28 (1995) 2693

[86] J.O. Rosas-Ortiz, J. Phys. A 29 (1996) 3281

[87] T. Fukui, N. Aizawa, Phys. Lett. A 180 (1993) 308

[88] V. Spiridonov, Phys. Rev. A 52 (1995) 1909

[89] M.S. Kumar, A. Khare, Phys. Lett. A 217 (1996) 73

[90] H.C. Fu, R. Sasaki, J. Phys. A 29 (1996) 4049

[91] V.G. Bagrov, B.F. Samsonov, J. Phys. A 29 (1996) 1011

[92] V.M. Eleonsky, V.G. Korolev, Phys. Rev. A 55 (1997) 2580

[93] S. Seshadri, V. Balakrishnan, S. Lakshmibala, J. Math. Phys. 39 (1998) 838

[94] F. Cannata, G. Junker, J. Trost, in Particles, fields and gravitation, J. Rembielinski Ed., AIP Conf. Proc. 453, Woodbury (1998) 209

[95] P.B. Abraham, H.E. Moses, Phys. Rev A 22 (1980) 1333

[96] N. Fernández-García, Derivación simplificada de la mecánica cuántica supersimétrica de segundo orden y algunas aplicaciones, B.Sc. Thesis, ESFM-IPN, Mexico (2004)

[97] J.F. Cariñena, A. Ramos, D.J. Fernández, Ann. Phys. 292 (2001) 42

[98] B.F. Samsonov, Phys. Lett. A 263 (1999) 274

[99] D.J. Fernández, B. Mielnik, O. Rosas-Ortiz, B.F. Samsonov, Phys. Lett. A 294 (2002) 168 
[100] D.J. Fernández, B. Mielnik, O. Rosas-Ortiz, B.F. Samsonov, J. Phys. A 35 (2002) 427

[101] D.J. Fernández, E. Salinas-Hernández, J. Phys. A 36 (2003) 2537

[102] D.J. Fernández, R. Muñoz, A. Ramos, Phys. Lett. A 308 (2003) 11

[103] O. Rosas-Ortiz, R. Muñoz, J. Phys. A 36 (2003) 8497

[104] R. Muñoz, Complex supersymmetrical transformations and exactly-solvable potentials, Ph.D. Thesis, Cinvestav, Mexico (2004)

[105] A. Ramírez, B. Mielnik, Rev. Mex. Fis. 49 S2 (2003) 130

[106] C. Quesne, N. Vansteenkiste, Helv. Phys. Acta 72 (1999) 71

[107] A. Andrianov, F. Cannata, M. Ioffe, D. Nishnianidze, Phys. Lett. A 266 (2000) 341

[108] R. Dutt, A. Gangopadhyaya, C. Rasinariu, U.P. Sukhatme, Phys. Rev. A 60 (1999) 3482

[109] A.P. Veselov, A.B. Shabat, Funct. Anal. Appl. 27 (1993) 81

[110] A.O. Barut, L. Girardello, Commun. Math. Phys. 21 (1971) 41

[111] W.M. Zhang, D.H. Feng, R. Gilmore, Rev. Mod. Phys. 62 (1990) 867

[112] J.R. Klauder, B.S. Skagerstam, Coherent states. Applications in physics and mathematical physics, World Scientific, Singapore (1985)

[113] A. Perelomov, Generalized coherent states and their applications, Springer, Berlin (1986)

[114] J. Beckers, N. Debergh, J. Math. Phys. 30 (1989) 1732

[115] S.M. Chumakov, A.B. Klimov, C. Saavedra, Phys. Rev. A 52 (1995) 3153

[116] A.M. El Gradechi, L.M. Nieto, Commun. Math. Phys. 175 (1996) 521

[117] H. Moya-Cessa, S. Wallentowitz, W. Vogel, Phys. Rev. A 59 (1999) 2920

[118] J.M. Sixdeniers, K.A. Penson, A.I. Solomon, J. Phys. A 32 (1999) 7543

[119] J.M. Sixdeniers, K.A. Penson, J.R. Klauder, Int. J. Mod. Phys. B 15 (2001) 4231

[120] V.V. Dodonov, J. Opt. B 4 (2002) R1

[121] O.I. Marichev, Handbook of integral transforms of higher transcendental functions, Ellis Horwood Ltd., England (1983)

[122] H. Bateman, Tables of integral transforms Vol. 1, A. Erdélyi Ed., McGraw Hill, New York (1954)

[123] D.J. Fernández, J. Negro, L.M. Nieto, Phys. Lett. A 324 (2004) 139 